\title{
Patient-specific parameter estimation
}

Coupling a heart model and experimental data

Domogo, Andrei A.; Ottesen, Johnny T.

Published in:

Journal of Theoretical Biology

DOI:

10.1016/j.jtbi.2021.110791

Publication date:

2021

Document Version

Early version, also known as pre-print

Citation for published version (APA):

Domogo, A. A., \& Ottesen, J. T. (2021). Patient-specific parameter estimation: Coupling a heart model and experimental data. Journal of Theoretical Biology, 526, [110791]. https://doi.org/10.1016/j.jtbi.2021.110791

\section{General rights}

Copyright and moral rights for the publications made accessible in the public portal are retained by the authors and/or other copyright owners and it is a condition of accessing publications that users recognise and abide by the legal requirements associated with these rights.

- Users may download and print one copy of any publication from the public portal for the purpose of private study or research.

- You may not further distribute the material or use it for any profit-making activity or commercial gain.

- You may freely distribute the URL identifying the publication in the public portal.

\section{Take down policy}

If you believe that this document breaches copyright please contact rucforsk@kb.dk providing details, and we will remove access to the work immediately and investigate your claim. 


\section{Highlights}

Patient-specific parameter estimation: coupling a heart model and experimental data

Andrei A. Domogo, Johnny T. Ottesen

- Mathematical modeling of the atrium, ventricle, veins, and arteries is given

- Unsteady Bernoulli equation is used for atrial inflow

- Model parameters are determined through patient-specific data

- Age and sex related differences in heart functions are captured

- Effects of dobutamine and glycopyrrolate to the cardiovascular system is quantified 


\title{
Patient-specific parameter estimation: coupling a heart model and experimental data
}

\author{
Andrei A. Domogo ${ }^{\mathrm{a}, \mathrm{b}}$, Johnny T. Ottesen ${ }^{\mathrm{b}, *}$ \\ ${ }^{a}$ University of the Philippines Baguio, Baguio City, Philippines \\ ${ }^{b}$ Roskilde University, Roskilde, Denmark
}

\begin{abstract}
This study develops a hemodynamic model involving the atrium, ventricle, veins, and arteries that can be calibrated to experimental results. It is a Windkessel model that incorporates an unsteady Bernoulli effect in the blood flow to the atrium. The model is represented by ordinary differential equations in terms of blood volumes in the compartments as state variables and it demonstrates the use of conductance instead of resistance to capture the effect of a non-leaking heart valve. The experimental results are blood volume data from 20 young (half of which are women) and 20 elderly (half of which are women) subjects during rest, inotropic stress (dobutamine), and chronotropic stress (glycopyrrolate). The model is calibrated to conform with data and physiological findings in 4 different levels. First, an optimization routine is devised to find model parameter values that give good fit between the model volume curves and blood volume data in the atrium and ventricle. Patient-specific information are used to get initial parameter values
\end{abstract}

\footnotetext{
*Corresponding author

Email addresses: aadomogo1@up.edu.ph (Andrei A. Domogo), johnny@ruc.dk (Johnny T. Ottesen)
} 
as a starting point of the optimization. Also, model pressure curves must show realistic behavior. Second, parametric bootstrapping is performed to establish the reliability of the optimal parameters. Third, statistical tests comparing mean optimal parameter values from young vs elderly subjects and women vs men are examined to support and present age and sex related differences in heart functions. Lastly, statistical tests comparing mean optimal parameter values from resting condition vs pharmacological stress are studied to verify and quantify the effects of dobutamine and glycopyrrolate to the cardiovascular system.

Keywords: cardiovascular dynamics modeling, patient-specific, parameter estimation, intropic stress, chronotropic stress

\section{Introduction}

Cardiovascular diseases may be fatal. The World Health Organization reported that Ischaemic heart disease and stroke have been the leading cause of death worldwide in 2016 and for the previous 15 years (WHO). Other heart diseases like stenosis, leaking heart valves, scar tissue in the ventricle, etc. are also common. Most of these heart diseases are diagnosed late, usually only when the ventricle starts to inefficiently pump blood. However, the majority of cardiovascular diseases can be interpreted in terms of pressure variation in the heart and blood vessels. Thus, understanding the blood flow in the cardiovascular system, i.e., looking at the volume and pressure profiles in the heart chambers, lungs, and blood vessels, may lead to earlier detection of these heart diseases and or their symptoms. Medical doctors believe that early signs of heart diseases can be seen in the atria (Douglas 
(2003); Aurigemma et al. (2009); Vieira et al. (2014)). Thus, in particular, the importance of studying atrial dynamics.

Mathematical modeling of the Cardiovascular system has progressed over the years. There are already thorough studies involving the interaction and dynamics of the cardiovascular system and its control (Danielsen and Ottesen (1997); Ottesen (1997); Ursino (1998); Ottesen and Olufsen (2011); Olufsen and Ottesen (2013); de los Reyes et al. (2014); Calderon et al. (2017)). There are even 3 dimensional models for blood flow in the heart (Peskin and Mcqueen (1989); Mcqueen and Peskin (1989)). These Cardiovascular models differ in the sense that some have a lot of parameters to achieve better physiological accuracy and some have lesser parameters to be able to compare and validate results with experimental data. Some studies that favoured complexity are Lu et al. (2001), Fan and Khoo (2002), Olufsen et al. (2005), and Lim et al. (2013). On the other hand, some of the studies that favoured comparability with data are Ottesen and Danielsen (2003), Pope et al. (2011), Aboelkassem et al. (2015), Marquis et al. (2018), and Williams et al. (2019). Of these models, only a few involved the atrium and only a few did patientspecific parameter estimation. To the best of our knowledge, there are no studies involving patient-specific parameter estimation during resting condition and pharmacological stress.

For this study, we will use a pulsatile cardiovascular model, involving the atrium, ventricle, veins, and arteries. We will build on the work of Ottesen (2011b) and Vingaard and Ottesen (2017) on an open loop model of blood flow in the left heart, denoted the Open Differential Model (See Figure 1). Linear pressure-flow relation (Windkessel approach) originally used in the 
Open Differential Model will be substituted by a second order relation obtained by the unsteady Bernoulli equation. There are available data for blood volume and flow in the left heart at rest and during pharmacological stress. These data were obtained from 20 young and 20 elderly subjects through Cardiac Magnetic Resonance Imaging by Rigshospitalet, Copenhagen, Denmark. Description of these phasic volume changes are given in the paper of Ahtarovski et al. (2012). The model and patient-specific data will be used to find individual system parameter values through optimization of fit in blood volume model curves. These model parameters can be used to estimate a normal range of values for the different parameters involved in blood circulation. Analysis of parameter values beyond and below the normal range may identify various pathophysiological conditions. Hence, the model and the data may be used to formulate specific interventions. 

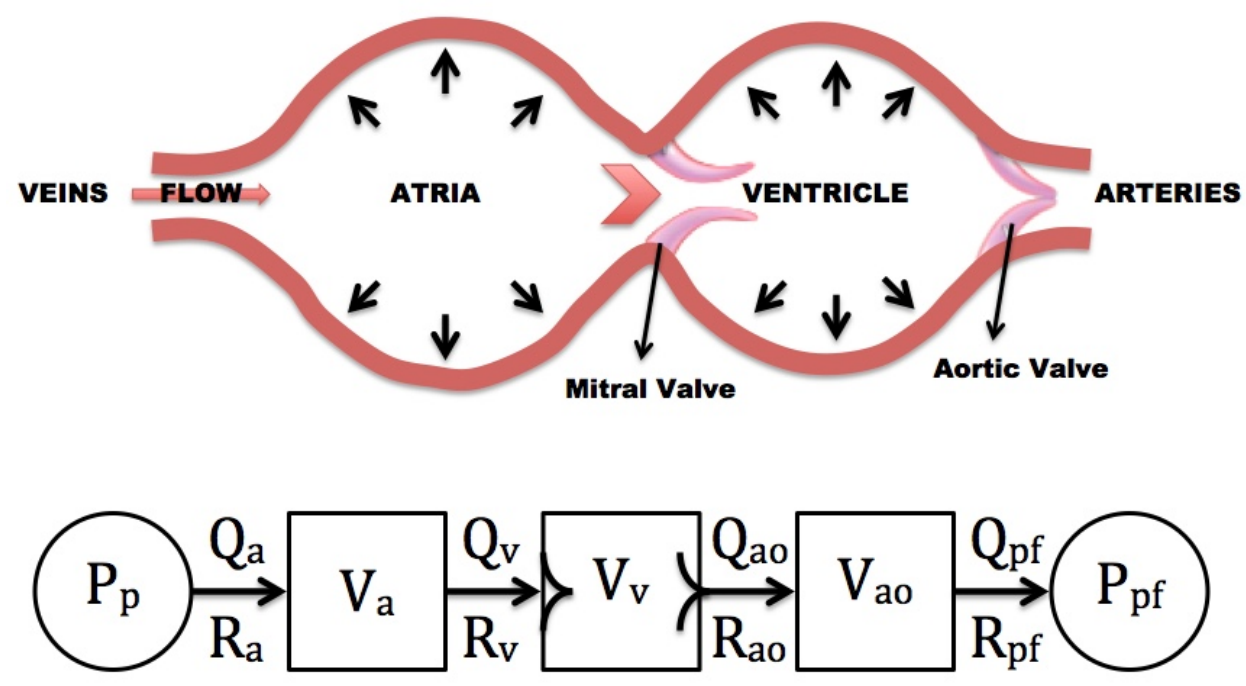

Figure 1: Top Panel: Physiological illustration of the left heart. Bottom Panel: In the open circuit model diagram, $V, Q, R$, and $P$ stand for volume, flow, resistance, and pressure, respectively. The subscripts $p, a, v, a o$, and $p f$ are for preload, atrium, ventricle, aorta, and peripherals, respectively.

Our main concerns for this study are the following. First, we observe whether a pulsatile Cardiovascular model involving the atria, ventricle, veins, and arteries can be developed. We ask. Is it possible to use the unsteady Bernoulli equation to represent blood flow into the atrium? Is it viable to use conductance instead of resistance to represent non-leaking heart valves? Is it useful to have blood volumes in compartments as state variables? Second, we see whether an optimization scheme can result in a good fit between model blood volume curves and blood volume data in the atria and ventricle during rest and pharmacological stress. We ask. To what extent can we use patient-specific data to get model parameter values that will serve as initial guess for the optimization scheme? Will the optimization scheme give 
optimal patient-specific model parameter values that are within physiological range? Will the optimal model parameters give rise to realistic blood flow dynamics in the atria, ventricle, veins, and arteries? Third, we perform a bootstrapping method to look at the reliability of the parameter estimates. We ask. Can we characterize the parameter uncertainty brought about by noise in data or perturbation of initial parameter estimates? Fourth, we discern whether a statistical test on the optimal model parameters comparing the mean values between young vs elderly and women vs men is in accordance with medical studies. We ask. What age and sex related differences in blood flow dynamics can be obtained from the model parameter values? Lastly, we inspect whether a statistical test on the optimal model parameters comparing the mean values between resting condition and pharmacological stress is able to characterize the effect of the drugs. We ask. What parameters are affected by the drugs? Are we able to quantify these effects?

\section{Methods}

This study undertakes a simple heart model involving the atrium that can be fitted well to blood volume data in the left heart. In this section, we describe how we are able to compute patient-specific model parameter values. We start by looking at the data used for parameter estimation, followed by the construction of the model. After which, we look at the optimization process used in the parameter estimation.

\subsection{Data}

Data for blood volume in the left heart and flow velocity out of the ventricle at rest and during pharmacological stress is from Rigshospital in Den- 
mark. Twenty young (20-30 years old) and 20 elderly (60-70 years) subjects with no known cardiovascular diseases or medication are considered. In each group, half are males and half are females. Blood volumes in the left atria and left ventricle, and flow velocity out of ventricle into aorta were calculated from 18-22 cross sectional images for 25 instance of one heart cycle. These images were acquired via Cardiac Magnetic Resonance Imaging or CMRi scanning. Heart rate of the participants were also measured. All these data were gathered during resting conditions and during pharmacological stress. Pharmacological stress was introduced through intravenous infusion of dobutamine (Dobutrex) and intravenous bolus injection of glycopyrrolate (Robinul). For the rest of the paper, when we use dobutamine and glycopyrrolate, we emphasize that we are referring specifically to the drugs Dobutrex and Robinul, respectively. Dobutamine was used to induce inotropic stress while glycopyrrolate was used to induce chronotropic stress. Roughly, pharmacological stress is used to simulate the effect of exercise to the heart and/or to assess heart functions (Travin and Wexler (1999); Marwick (2003)). In particular, inotropic stress increases the heart's power to pump blood or contractility and chronotropic stress increases heart rate (Danielsen (1998)). Further description of the data can be found in the paper of Ahtarovski et al. (2012).

\subsection{Model Formulation}

The part of departure is a compartmental model of blood flow in the left heart by Vingaard and Ottesen (2017). In section 2.2.1 and 2.2.2, we give specific details about the model as given in Vingaard and Ottesen (2017). We then introduce a modification of the model in section 2.2.3. The model is de- 
scribed by the following equations, which is denoted as the Open Differential Model.

$$
\left\{\begin{array}{l}
\dot{V}_{a}=Q_{a}-Q_{v} \\
\dot{V}_{v}=Q_{v}-Q_{a o} \\
\dot{V}_{a o}=Q_{a o}-Q_{p f} \\
\dot{Q}_{a}=\frac{1}{L_{a}}\left[\left(P_{p}-P_{a}\right)-R_{a} \cdot Q_{a}\right] \\
\dot{Q}_{v}=\frac{1}{L_{v}}\left[\left(P_{a}-P_{v}\right)-R_{v} \cdot Q_{v}\right] \\
\dot{Q}_{a o}=\frac{1}{L_{a o}}\left[\left(P_{v}-P_{a o}\right)-R_{a o} \cdot Q_{a o}\right] \\
Q_{p f}=\frac{1}{R_{p f}}\left(P_{a o}-P_{p f}\right) \\
P_{a}=\frac{1}{C_{a}}\left(V_{a}-V_{a, u}\right) \\
P_{v}=\frac{1}{C_{v}}\left(V_{v}-V_{v, u}\right) \\
P_{a o}=\frac{1}{C_{a o}}\left(V_{a o}-V_{a o, u}\right)
\end{array}\right.
$$

where, $V, Q, P, R, L$, and $C$ stand, respectively, for volume, flow, pressure, resistance, inertance, and compliance. The subscripts $p, a, v, a o$, and $p f$ refer to preload (veins), atrium, ventricle, aorta, and peripheral (capillaries), respectively. Also, the subscript $u$ pertains to the unstressed volume in a given compartment.

These equations are derived from the following natural relations. (1) Law of conservation: $\dot{V}=Q_{\text {in }}-Q_{\text {out }}$. The change in volume in a compartment is equal to the difference of the inflow and outflow in the compartment. (2) The total pressure difference is assumed to be caused partly by friction due to the blood's viscosity and partly by the effect of inertia: $P_{\text {in }}-P_{\text {out }}=R \cdot Q+L \cdot \dot{Q}$. (3) Blood vessel compliance: $V=C \cdot P+V_{u}$. The volume in a compartment 
is an increasing function of pressure. This relation is assumed to be linear. The pressure $P$ denotes the transmural pressure, that is, it is the pressure in the blood vessel relative to the ambient pressure. Note that the volume in the blood vessels is equal to the unstressed volume when the transmural pressure is zero.

\subsubsection{Variable Resistance}

In order to take account of the heart valves, a variable resistance is introduced for the flow into and out of the ventricle. To avoid infinite quantities, these are expressed in term of conductivities, $\rho$, which is the reciprocal of resistance. That is, $R=1 / \rho$. The mitral and aortic valves open and close as a result of the pressure difference between adjacent compartments. This means that respective conductivities are also dependent on these pressure differences. It is assumed that the conductivities can be described in the following way. For the mitral valves,

$$
\rho_{v}=\left(\rho_{v M}-\rho_{v m}\right) \cdot \frac{1}{1+e^{-k\left(P_{a}-P_{v}\right)}}+\rho_{v m}
$$

and for the aortic valves,

$$
\rho_{a o}=\left(\rho_{a o M}-\rho_{a o m}\right) \cdot \frac{1}{1+e^{-k\left(P_{v}-P_{a o}\right)}}+\rho_{a o m}
$$

where, $\rho_{v m}$ and $\rho_{v M}$ are respectively the minimum (closed valve) and maximum (open valve) conductivity for the flow into the ventricle. Correspondingly, $\rho_{a o m}$ and $\rho_{a o M}$ are the conductivity for the flow out of the ventricle into the aorta. The constant $k$ determines how fast the valves open and close. For both valves, the minimum conductivity is equal to zero. This corresponds to having an infinite resistance when the valves are closed. 
For the resistance to the flow into the atrium, there is a very asymmetric spatial geometry for flow between veins and atria. Hence it seems that the relative slow flow from veins to atria is easier than that of the fast back flow from atria to veins during contractions. Slow flow causes less resistance and inertance than fast flow. Also, the 90 degree change in direction of the impulse, which is pictured in Figure 2, may follow the same pattern. In addition, one may imagine that a part of the back flow from atria to veins is reflected at the opposite vessel wall. To account for such asymmetry, we model the resistance to flow into the atrium also to be pressure dependent. That is, the conductivity for the flow into the atrium is given by the following.

$$
\rho_{a}=\left(\rho_{a M}-\rho_{a m}\right) \cdot \frac{1}{1+e^{-k\left(P_{p}-P_{a}\right)}}+\rho_{a m}
$$

Thus, the resistances for flow into the atrium, ventricle, and aorta in system (1) is state dependent. That is, $R_{a}=1 / \rho_{a}, R_{v}=1 / \rho_{v}$, and $R_{a o}=1 / \rho_{a o}$ and $\rho_{v}=\rho_{v}\left(P_{a}, P_{v}\right), \rho_{a o}=\rho_{a o}\left(P_{v}, P_{a o}\right)$, and $\rho_{a}=\rho_{a}\left(P_{p}, P_{a}\right)$, which is given by $(2),(3)$, and (4) respectively. In the conductivities given in (2), (3), and (4), $k$ determines how fast the valves transition between opening and closing. Since there are no available data on differences between these transitions for the different heart valves, we simply let $k$ be equal for all the valves to minimize the number of model parameters. 

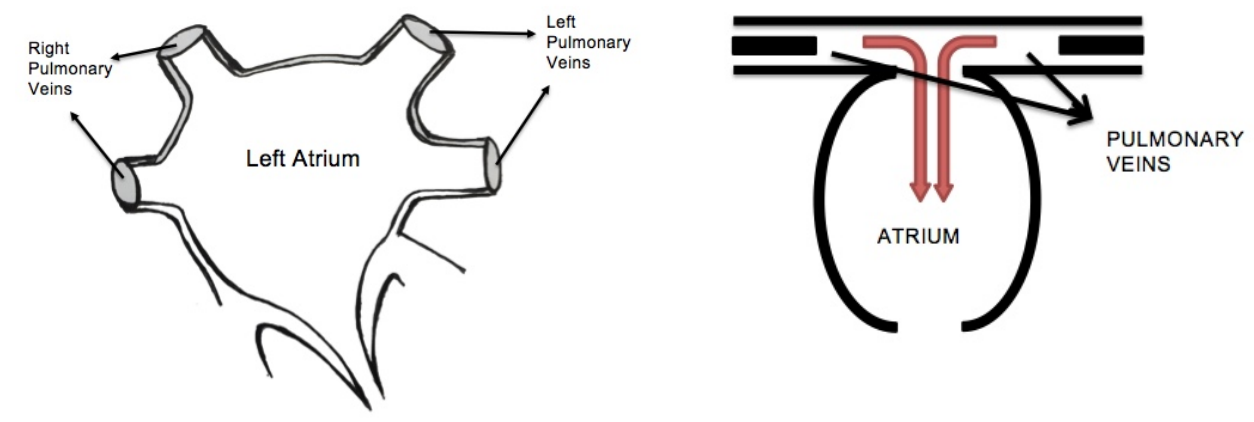

Figure 2: Asymmetric spatial geometry for flow between veins and atria.

\subsubsection{The Pumping Heart}

It is also assumed that the compliance in the atrium and ventricle are functions of time. This is sensible since the two compartments expand and contract significantly during each heartbeat. The representation of a time dependent compliance for the ventricle is taken from Ellwein et al. (2009). The time dependent compliance for the atrium is modeled similarly. The compliance is often given in terms of its reciprocal, which is elastance. In the following, $e_{v}$ represents the elastance of the ventricle, while $e_{v m}$ and $e_{v M}$ denote the minimum and maximum elastance of the ventricle respectively. A similar notation is given for the atrial elastance. We have the following equations.

$$
e_{v}(t)= \begin{cases}e_{v m}+\frac{1}{2}\left(e_{v M}-e_{v m}\right)\left(1-\cos \left(\frac{\pi t}{T_{v s}}\right)\right) & 0 \leq t \leq T_{v s} \\ e_{v m}+\frac{1}{2}\left(e_{v M}-e_{v m}\right)\left(1+\cos \left(\frac{\pi\left(t-T_{v s}\right)}{T_{v r}}\right)\right) & T_{v s} \leq t \leq T_{v s}+T_{v r} \\ e_{v m} & T_{v s}+T_{v r} \leq t \leq T\end{cases}
$$




$$
e_{a}(t)= \begin{cases}e_{a m}+\frac{1}{2}\left(e_{a M}-e_{a m}\right)\left(1-\cos \left(\frac{\pi t}{T_{a s}}\right)\right) & 0 \leq t \leq T_{a s} \\ e_{a m}+\frac{1}{2}\left(e_{a M}-e_{a m}\right)\left(1+\cos \left(\frac{\pi\left(t-T_{a s}\right)}{T_{a r}}\right)\right) & T_{a s} \leq t \leq T_{a s}+T_{a r} \\ e_{a m} & T_{a s}+T_{a r} \leq t \leq T\end{cases}
$$

The constants $T_{v s}$ and $T_{v r}$ correspond to the time when the ventricle assumes its maximum and minimum elastance, respectively. A corresponding notation is used for the atrium. Heart rate is taken into account as $T_{v s}, T_{v r}, T_{a s}$, and $T_{a r}$ are calculated as fractions of the period of a heartbeat, denoted by $T$. These fractions are denoted as $T_{v s, f r a c}, T_{v r, f r a c}, T_{a s, f r a c}$, and $T_{a r, f r a c}$, respectively. During implementation, a parameter DT is introduced to shift the curve for the elastance of the atrium to the left. That is, DT accounts for the gap between the contraction of the atrium and the ventricle and we define $D T_{\text {frac }}:=D T / T$. A visualization of a time series profile of the elastance of the atrium and ventricle for one heart cycle is given in Figure 3.

The compliance of the atrium and ventricle in system (1) are then time dependent, $C_{a}=1 / e_{a}$ and $C_{v}=1 / e_{v}$, with elastances $e_{a}=e_{a}(t)$ and $e_{v}=$ $e_{v}(t)$ as given in (5) and (6), respectively. 


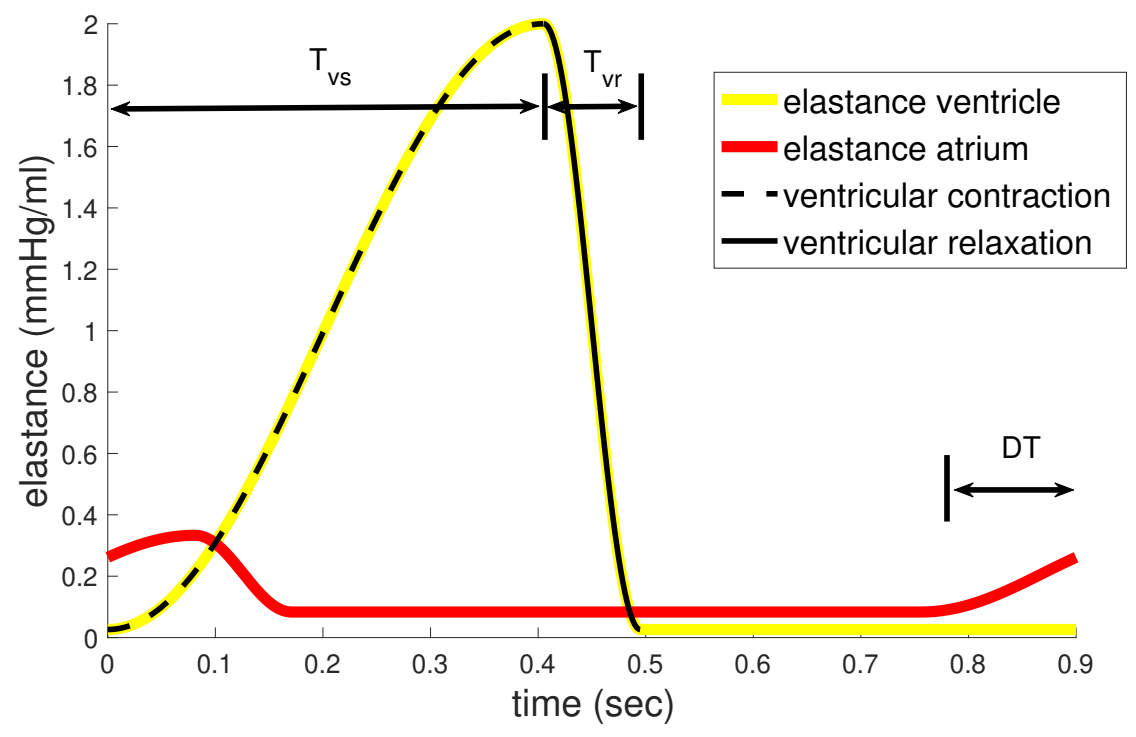

Figure 3: Elastance curve profile of the atrium (red curve) and ventricle (yellow curve) in one heart cycle.

\subsubsection{The Atrial Inflow}

We modify the model by changing the equation for flow into the atrium, $Q_{a}$, using the unsteady Bernoulli equation. We hope that modifying the model will impact the behaviour of the curves for the volume, pressure, and flow positively. The flow from the pulmonary veins to the atria using the unsteady Bernoulli equation is given in equation (7), see Grimes et al. (1995).

$$
P_{p}-P_{a}=\rho L_{e f f} \frac{d u_{a}}{d t}+\frac{\rho u_{a}^{2}}{2}
$$

where, $P_{p}$ and $P_{a}$ stands for venous and left atrial pressure, respectively, $u_{a}$ is the velocity at the atrial inlet, $L_{e f f}$ is the length of the summated pulmonary veins, and $\rho$ is the blood density. Note, here flow is assumed uni-directional. Since the pulmonary veins leading to the atria is treated as a single tube, 
the flow into the atria, $Q_{a}$, is equal to $A_{a} \cdot u_{a}$, where $A_{a}$ is the cross sectional area of the tube. Incorporating this into equation (7), we get

$P_{p}-P_{a}=\rho L_{e f f} \frac{d\left(Q_{a} / A_{a}\right)}{d t}+\frac{\rho\left(Q_{a} / A_{a}\right)^{2}}{2}=\frac{\rho L_{e f f}}{A_{a}} \frac{d\left(Q_{a}\right)}{d t}+\frac{\rho}{2 A_{a}^{2}} Q_{a}^{2}=L_{a} \frac{d\left(Q_{a}\right)}{d t}+b_{a} Q_{a}^{2}$,

where $b_{a}:=\frac{\rho}{2 A_{a}^{2}}$ and the inertance, $L_{a}=\frac{\rho L_{e f f}}{A_{a}}$. Thus, the flow from the veins into the atria is described by

$$
\dot{Q}_{a}=\frac{1}{L_{a}}\left[\left(P_{p}-P_{a}\right)-b_{a} Q_{a}^{2}\right]
$$

To take account for flow in the opposite direction from the atria into the veins, the last sign changes. Thus, the general equation for flow between veins and atria becomes,

$$
\dot{Q}_{a}=\frac{1}{L_{a}}\left[\left(P_{p}-P_{a}\right)-b_{a}\left|Q_{a}\right| Q_{a}\right]
$$

Note, equation (9) corresponds to assuming $R_{a}$ in system (1) is flow dependent, that is, $R_{a}=b_{a}\left|Q_{a}\right|$. The formulation of $Q_{a}$ using the unsteady Bernoulli equation makes it possible to omit the ad-hoc formulation of variable resistance for blood flow into the atrium given by equation (4).

As a denotation, we call system (1) together with the details and modification given in sections 2.2.1, 2.2.2, 2.2.3 as the Open Bernoulli-Differential Model. For this model, we have the following 25 model parameters. 1) Unstressed volumes: $V_{a, u}, V_{v, u}$, and $\left.V_{a o, u}, 2\right)$ Compliances: $C_{a o}$ and the elastance and time constant parameters $e_{a m}, e_{a M}, e_{v m}, e_{v M}, T_{v s}, T_{v r}, T_{a s}, T_{a r}$, and $D T$, which arise from the time dependence of compliance of the atria and ventricle, 3) Constant Pressures: $P_{p}$ and $\left.P_{p f}, 4\right)$ Resistances: $R_{p f}$, and the conductance 
parameters $\rho_{v m}, \rho_{v M}, \rho_{a o m}, \rho_{a o M}$, and valve constant $k$, which arise from the variable resistance to flow into the ventricle and aorta, 5) Inertances: $L_{a}, L_{v}$, and $L_{a o}$, and 6) Bernoulli parameter: $b_{a}$.

\subsection{Parameter Estimation}

The objective of this section is to find parameter values that will give atrial and ventricular volume curves that conforms with given data. It is also required to have flow and pressure curves that show realistic behavior. The parameter estimation process involves (1) an a priori parameter estimation followed by (2) an iterated optimization.

In the a priori estimation, available data are used to calculate parameter values for the model. Also, parameter values from related literature are used for parameter values that cannot be calculated from available data. For the optimization, we use the a priori parameter values as initial condition for a minimization problem, which we solve in Matlab 2020a using the fmincon function. The objective cost of this minimization problem is given by

$$
\begin{aligned}
\text { Obj. Cost } & =\frac{1}{W T} \sum_{i=0}^{24}\left[\left\{\frac{V_{a}\left(t_{i}\right)-V_{a}^{*}\left(t_{i}\right)}{V_{a}^{*}\left(t_{i}\right)}\right\}^{2}+\left\{\frac{V_{v}\left(t_{i}\right)-V_{v}^{*}\left(t_{i}\right)}{V_{v}^{*}\left(t_{i}\right)}\right\}^{2}\right] \\
& +\frac{W}{W T} \sum_{i \in I}\left\{\frac{V_{a}\left(t_{i}\right)-V_{a c}^{*}\left(t_{i}\right)}{V_{a c}^{*}\left(t_{i}\right)}\right\}^{2}+\frac{W}{W T} \sum_{i \in J}\left\{\frac{V_{v}\left(t_{i}\right)-V_{v c}^{*}\left(t_{i}\right)}{V_{v c}^{*}\left(t_{i}\right)}\right\}^{2} \\
& +10^{6} \sum_{i=0}^{24}\left(\frac{P_{a o}\left(t_{i}\right)-100}{100}\right)^{2} \quad \text { if } \quad P_{a o}\left(t_{i}\right)<70 \text { or } P_{a o}\left(t_{i}\right)>130 \\
& +10^{6} \sum_{i=0}^{24}\left(\frac{P_{v}\left(t_{i}\right)-100}{100}\right)^{2} \text { if } \quad P_{v}\left(t_{i}\right)>130 \\
& +10^{6} \sum_{i=0}^{24}\left(\frac{Q_{p f}\left(t_{i}\right)-125}{125}\right)^{2} \quad \text { if } \quad Q_{p f}\left(t_{i}\right)<0 \text { or } Q_{p f}\left(t_{i}\right)>250 .
\end{aligned}
$$

The first line of the objective cost function is the sum of the squares of the relative error between volume in the atrium and ventricle from model simulation and from 25 data points. The volume from model simulation are 
given by $V_{a}\left(t_{i}\right)$ and $V_{v}\left(t_{i}\right)$ while the volume from data are given by $V_{a}^{*}\left(t_{i}\right)$ and $V_{v}^{*}\left(t_{i}\right)$. The $t_{i}^{\prime} s$ are 25 equally spaced time instances from 0 to $T$ (duration of a heart cycle for a given person). The second line in the objective cost function is added to give more weight to visually identified important data points. The important data points are initially where the data have some qualitative change in behavior. Example of important data points are points where there are local extrema, change in concavity, and endpoints. After optimization, important points are added or removed when we want to influence the simulated curves to move in certain directions. After which, we perform again the optimization. The important data points from volume data in the atrium and ventricle are denoted by $V_{a c}^{*}\left(t_{i}\right)$ and $V_{v c}^{*}\left(t_{i}\right)$, respectively. $I$ and $J$ are correspondingly the index sets containing the index of the important data points for the atrium and ventricle. We let $n$ be the number of elements of $I$ and $k$ be the number of elements of $J . W$ is an assigned weight and $W T=W \times(n+k)+50$. This makes the weight of the relative error between volume in the atrium and ventricle 1 in total. The last 3 terms in the objective cost function are penalty terms so that pressure in the aorta stays between $70 \mathrm{mmHg}$ and $130 \mathrm{mmHg}$, pressure in the ventricle is below $130 \mathrm{mmHg}$, and flow in the peripherals is between 0 and $250 \mathrm{ml} / \mathrm{s}$. The value of the weight $W$ and the set of important points $I$ and $J$ for each of the 40 persons is given in Supplementary A.

\subsubsection{A Priori Parameter Estimation}

We put together information from literature and patient-specific data to get the a priori model parameters. Atrial and ventricular volume data from each person are used to get the a priori volume parameter values. However, 
pressure data in the compartments were not measured for these persons. Hence, average values from the literature are utilized. A lot of a priori parameters are dependent on a persons total blood volume, $V_{\text {Total }}$. The total blood volume is calculated using the following formula that uses the body surface area (BSA) and sex as input (Shoemaker (1989)).

$$
V_{\text {Total }}= \begin{cases}(3.47 \cdot B S A-1.954) \cdot 1000 \mathrm{ml}, & \text { woman } \\ (3.29 \cdot B S A-1.229) \cdot 1000 \mathrm{ml}, & \text { man }\end{cases}
$$

To get BSA, we use Boyd's formula (Boyd (1935)), BSA $=0.03330 \cdot w^{0.6157-0.0188 \cdot \log (w)}$. $H^{0.3}$, where $w$ is the weight in kilograms and $H$ is the height in centimeters.

Unstressed Volume. The unstressed volume in the compartments are taken as percentages of the volume in the compartments and the volume in the compartments are percentages of $V_{\text {Total }}$ (see Table 1 ).

\begin{tabular}{|l|l|l|l|}
\hline Parameter & Formula & Units & Source \\
\hline$V_{a, u}$ & $\begin{array}{l}33.3 \% \cdot V_{a} \\
\text { where } V_{a}=1.745 \% \cdot V_{\text {Total }}\end{array}$ & $\mathrm{ml}$ & $\begin{array}{c}\text { Vingaard and Ottesen (2017) } \\
\text { Guyton and Hall (2016) }\end{array}$ \\
\hline$V_{v, u}$ & $\begin{array}{l}9.1 \% \cdot V_{v} \\
\text { where } V_{v}=2.0026 \% \cdot V_{\text {Total }}\end{array}$ & $\mathrm{ml}$ & $\begin{array}{c}\text { Vingaard and Ottesen (2017) } \\
\text { Guyton and Hall (2016) }\end{array}$ \\
\hline$V_{a o, u}$ & $\begin{array}{l}74.4 \% \cdot V_{a o} \\
\text { where } V_{a o}=13 \% \cdot V_{\text {Total }}\end{array}$ & $\mathrm{ml}$ & $\begin{array}{c}\text { Vingaard and Ottesen (2017) } \\
\text { Guyton and Hall (2016) }\end{array}$ \\
\hline
\end{tabular}

Table 1: A priori values for unstressed volumes in the compartments.

Constant Pressures. The a priori estimates for preload pressure, $P_{p}=5.8$ $\mathrm{mmHg}$ and peripheral pressure, $P_{p f}=67.9 \mathrm{mmHg}$ are taken from Vingaard 
and Ottesen (2017) and Danielsen (1998), respectively.

Compliance. The compliance of a compartment is calculated through a reformulation of the linear relation of volume and pressure, i.e., $C=\frac{V-V_{u}}{P}$. The minimum and maximum atrial compliance is calculated using values taken in the end systolic phase and end diastolic phase, respectively (see Table 2). The end systolic and diastolic volumes can be identified from blood volume data for the atrium and ventricle. In general, the end systolic volumes are the minimum blood volume data while the end diastolic volume is the maximum blood volume data. Similarly for the minimum and maximum ventricular compliance.

\begin{tabular}{|l|l|l|l|}
\hline Parameter & Formula & Units & Source \\
\hline$C_{a m}$ & $\frac{V_{a, E S V}-V_{a, u}}{P_{a, E S V}}$ & $\mathrm{ml} / \mathrm{mmHg}$ & Data and Blume et al. (2011) \\
$C_{a M}$ & $\frac{V_{a, E D V}-V_{a, u}}{P_{a, E D V}}$ & $\mathrm{ml} / \mathrm{mmHg}$ & Data and Blume et al. (2011) \\
$C_{v m}$ & $\frac{V_{v, E S V}-V_{v, u}}{P_{v, E S V}}$ & $\mathrm{ml} / \mathrm{mmHg}$ & Data and Guyton and Hall (2006) \\
$C_{v M}$ & $\frac{V_{v, E D V}-V_{v, u}}{P_{v, E D V}}$ & $\mathrm{ml} / \mathrm{mmHg}$ & Data and Guyton and Hall (2006) \\
$C_{a o}$ & $\frac{V_{a o}-V_{a o, u}}{P_{a o}}$ & $\mathrm{ml} / \mathrm{mmHg}$ & Data and Danielsen (1998) \\
\hline
\end{tabular}

Table 2: A priori values for minimum and maximum compliance of the compartments.

Resistance. The resistance to flow into a compartment is calculated through a reformulation of the relation of pressure difference and flow. In particular for open heart valves, we have that $R_{v m}=\frac{P_{a, \text { dias }}-P_{v, \text { dias }}}{Q_{a}}$ and $R_{a o m}=\frac{\left(P_{v}-P_{a o}\right)}{Q_{a o}}$ where $P_{v}>P_{a o}$. The peripheral resistance, $R_{p f}$, is equal to $\frac{P_{a o}-P_{p f}}{Q_{p f}}$. In Vingaard and Ottesen (2017), they have approximated these minimum resis- 
tances as the average change in pressures divided by the average flow. They have the following approximations: $P_{a, \text { dias }}-P_{v, \text { dias }} \approx 2 \mathrm{mmHg}, P_{v}-P_{a o} \approx 2$ mmHg, when $P_{v}>P_{a o}$, and $P_{a o}-P_{p f} \approx 18.8 \mathrm{mmHg}$. Also, the average flow was approximated by the total flow, $Q_{\text {Total }}:=\frac{V_{\text {Total }}}{60 \mathrm{~s}}$, assuming that the blood circulates within one minute. We adopt these approximations for the a priori values for resistances from which the a priori values of $\rho_{v M}$ and $\rho_{a M}$ follows (see Table 3). The minimum conductivities $\rho_{v m}$ and $\rho_{a m}$, which corresponds to the maximum resistance to flow (closed valves) respectively, are set to zero. The value of $k$, the parameter associated with how fast the valves open and close, is set at 1000 (Vingaard and Ottesen (2017)).

\begin{tabular}{|l|l|l|l|}
\hline Parameter & Formula & Units & \multicolumn{1}{|c|}{ Source } \\
\hline$R_{v m}$ & $\frac{2}{Q_{\text {total }}}$ & $\mathrm{mmHg} \cdot \mathrm{s} / \mathrm{ml}$ & Data and Vingaard and Ottesen (2017) \\
$R_{a o m}$ & $\frac{2}{Q_{\text {total }}}$ & $\mathrm{mmHg} \cdot \mathrm{s} / \mathrm{ml}$ & Data and Vingaard and Ottesen (2017) \\
$R_{p f}$ & $\frac{18.8}{Q_{\text {total }}}$ & $\mathrm{mmHg} \cdot \mathrm{s} / \mathrm{ml}$ & Data and Vingaard and Ottesen (2017) \\
\hline
\end{tabular}

Table 3: A priori values for resistance to flow into the compartments.

Time Constants. The time constants are visually identified from volume data in the atrium and ventricle. The time when the left atrium is contracting is given by $T_{a s}$. The initial value of $T_{a s}$, is taken as the number of volume data points during the atrial systole multiplied by one time step (the period $T$ divided by 25). $T_{a r}$ is the time when the left atrium achieves its relaxed state after contracting. To get the initial value, we count the number of volume data points after atrial systole that seem to be constant. Usually, the duration is one time step. $T_{v s}$ is the time when the left ventricle is contracting. The initial value is taken to be the number of volume data points during 
the ventricular systole multiplied by one time step $(T / 25) . T_{v r}$ is the time when the left ventricle achieves its relaxed state after contracting. To get this, we count the number of volume data points after ventricular ejection that seem to be constant. Usually, the duration is one or two time steps. DT is the time between the contraction of the atrium and the ventricle. We set it at $T_{a s}+T_{a r}$. Now, we hypothesized that the data might not be recorded exactly at the start of the isovolumetric contraction of the ventricle. So we introduce an additional parameter, $S$, to take into account this possible delay in the time of start for recording blood volume data. We set it at 1 or 2 time steps depending on the number of the volume data points that seem to be constant at the end diastolic phase. The a priori values of the time constants for each person is given in Supplementary A.

Inertance. The inertance parameters are taken from literature. From Danielsen (1998), we have that $L_{a}=5.0 \times 10^{-5} \mathrm{mmHg} \cdot \mathrm{s}^{2} / \mathrm{ml}$ and $L_{v}=$ $4.6 \times 10^{-4} \mathrm{mmHg} \cdot \mathrm{s}^{2} / \mathrm{ml}$. Also, $L_{a o}=2.1 \times 10^{-5} \mathrm{mmHg} \cdot \mathrm{s}^{2} / \mathrm{ml}$ is taken from Ottesen (2011b).

Bernoulli Parameter. The Bernoulli parameter, $b_{a}$, is given by the blood density divided by twice the square of the cross sectional area of the inlet leading to the atrium. For the cross sectional area of the inlet leading to the atrium, we use $8 \mathrm{~cm}^{2}$, which is the total area of the venae cavae (Guyton and Hall (2016)). With these, $b_{a}=\frac{1.06 \mathrm{~g} / \mathrm{cm}^{3}}{2\left(8 \mathrm{~cm}^{2}\right)^{2}}$. Multiplying the conversion factor $\frac{1}{1330}, b_{a}=6.23 \times 10^{-6} \mathrm{mmHg} \cdot s^{2} / \mathrm{ml}^{2}$. However, we found in the optimization that the a priori value of $b_{a}$ is a factor 30 greater during rest and a factor 10 
greater during pharmacological stress.

Initial Values. The initial values for the system of ODE for the Open Bernoulli-Differntial Model are given as follows. The initial value for volume in the atrium, $V_{a}(0)$, and ventricle, $V_{v}(0)$, are taken to be the first volume data in the atrium and ventricle, respectively. The initial value for volume in the aortic compartment, $V_{a o}(0)$, is assumed to be $10 \%$ of $V_{\text {Total }}$. The initial value for flow into the atrium, $Q_{a}(0)$, is calculated from model equations assuming that initially the ODE is in a steady state. We have that $Q_{a}(0)=\sqrt{\frac{1}{b_{a}} \cdot\left(P_{p}-\frac{V_{a, E S V}-V_{a, u}}{C_{a M}}\right)}$. The initial value for the flow into the ventricle, $Q_{v}(0)$, and flow into the aorta, $Q_{a o}(0)$ are assumed to be zero because at $t=0$, the heart valves are closed.

The a priori parameters for each of the 40 persons were calculated using the above formulas or values. They were used as an initial guess in the optimization process. For a few persons however, these initial values led to failure of the optimization process due to parameters having values beyond the boundary constraints during iterations in the optimization process. The boundary constraints were set to $50 \%$ above and below the initial parameter values. Also, for a few persons, these initial values led to having optimal parameters from undesired local minima. Due to these, we made adjustments to the corresponding a priori parameters. That is, we perturbed some of the parameters for persons where we did not achieve a desired fit in the volume curves. Also, we have re-adjusted some of the a priori parameters after comparing their values to the computed optimal parameters. During phar- 
macological stress, some of these parameter values where adjusted to take into account the effect of the drugs. A table of the final a priori parameters for resting condition and during pharmacological stress is given in Supplementary A. Furthermore, a description of the origin of the a priori parameter values taken from (Vingaard and Ottesen (2017)) is given in Supplementary A.1.

\subsubsection{Optimization}

In this section, the a priori parameters are used as initial point for an optimization scheme. We optimize all model parameters except for $k, \rho_{v m}$, $\rho_{a o m}, V_{a}(0), V_{v}(0), Q_{v}(0)$, and $Q_{a o}(0)$. The parameter $k$ is chosen as a fixed sufficiently large value. $\rho_{v m}$ and $\rho_{a o m}$ are chosen to be 0 , which corresponds to the maximum resistance $R_{v M}$ and $R_{a o M}$ being equal to infinity. The initial volumes in the atrium and ventricle, $V_{a}(0)$ and $V_{v}(0)$, are fixed values from data. The initial flow into the ventricle and into the aorta, $Q_{v}(0)$ and $Q_{a o}(0)$, are fixed to 0 because the heart is at the isovolumetric stage. Also, post optimization sensitivity analysis show that $k$ is not a sensitive parameter (see sensitivity analysis given in Supplementary F).

First, observe that the objective cost is a function of the parameter values and the volume and pressure output of the model. That is, if $X($ par $)$ is the volume and pressure output of the model given the input parameters par, we have that

$$
\text { Objective Cost }=f(\text { par, } X(\text { par })) \text {. }
$$

Our minimization problem is given by

$$
\min f(\text { par, } X(\text { par })) \quad \text { where } \quad \text { par } \in[0.5 * \text { init_par, } 1.5 * \text { init_par }] \text {. }
$$


We note that the initial parameters are the calculated a priori parameters, which are approximations. These values are away from the optimal values in different degrees. Hence, we want to choose bounds for the value of par such that our minimization problem will give desirable results. From simulations, we have observed that moving $50 \%$ above and below the initial parameters are suitable boundary values.

Now, to explore other minimizers near the obtained minimizer of $f$, we solve for the minimizer of $f$ iteratively using the optimal parameters as initial parameters for the next minimization problem. We have the following scheme.

Initialization:

init_par $=$ a priori parameters

obj_cost_old $=\infty$

opt_par $=$ arg_min of $f$, where par $\in[0.5 *$ init_par, $1.5 *$ init_par $]$

obj_cost $=f\left(o p t \_p a r, X\left(o p t \_p a r\right)\right)$

while $\left|o b j \_c o s t-o b j \_c o s t \_o l d\right| / o b j \_c o s t<t o l$ do:

$$
\begin{aligned}
& o b j \_c o s t \_o l d=o b j \_c o s t \\
& \text { init_par }=o p t \_p a r \\
& o p t \_p a r=a r g \_m i n \text { of } f, \text { where } p a r \in[0.5 * \text { init_par, } 1.5 * \text { init_par }] \\
& o b j \_c o s t=f\left(o p t \_p a r, X\left(o p t \_p a r\right)\right)
\end{aligned}
$$

We use the Matlab function fmincon to solve for the minimizer of $f$. In particular, we use the default Interior Point Algorithm. This option solves 
the minimization problem through a sequence of approximate minimization problems involving barrier functions. The approximate minimization problems are then solved by going in the direction of a direct(Newton) step in each iteration. If the Newton step fails, the algorithm goes into a Conjugate Gradient step using a trust region (The Mathworks, Inc.). We solve for the volume and flow output of the Model using a combination of the Forward and Backward Euler method and RK2 method. The Forward Euler is used to solve for the volume. We note that the change in flow into the ventricle and aorta makes the system of ODEs stiff. Hence, we use the backward Euler method, which is a more stable numerical solver, to solve for the flow into the ventricle and the aorta. We use the RK2 method to solve for the flow into the atrium. In the model, when a non-leaky valve closes the corresponding resistance to flow goes to infinity corresponding to conductivity zero. While the resistance approach infinity the corresponding flow through the valve approach zero. Thus, the product appearing on the right hand side of the ODEs in (1) need to be carefully calculated to avoid failure. Due to this, solving the ODE using explicit methods is not appropriate. This is remedied by the use of the Backward Euler method. A Backward Euler method was also used in solving a system of ODEs corresponding to a cardiovascular model in (Hoppensteadt and Peskin (2002)) to address a similar problem. The codes for the optimization and numerical ODE solver and a detailed explanation of the numerical method used are given in Supplementary E. 


\section{Results}

This section is written in the following order. First, we show the model curves that are obtained from optimal parameter values. Secondly, we present the range of values for the obtained optimal model parameters. Finally, we give the statistical findings about significant differences between mean values of optimal parameters from different subgroups of the data.

\subsection{Model Fit}

The simulated model curves show that the model is able to capture the dynamics of blood flow in the left heart. Through optimization, the model can be calibrated well to blood volume data at rest and during pharmacological stress. The sum of squared errors (SSE) between model curves and data is summarized in Figure 4. The mean \pm standard deviation of the SSE for the atrium at rest, dobutamine stress, and glycopyrrolate stress are $17.43 \pm 6.23$, $20.56 \pm 8.51,16.07 \pm 6.69$, respectively. The mean \pm standard deviation of the SSE for the ventricle at rest, dobutamine stress, and glycopyrrolate stress are $38.61 \pm 22.42,47.02 \pm 25.22,31.6296 \pm 13.55$, respectively. Using the SSE as basis, we see that the model is calibrated best during glycopyrrolate stress and least at dobutamine stress. Also, we observe that we are able to get a better fit in the blood volume in the atrium than in the ventricle. 


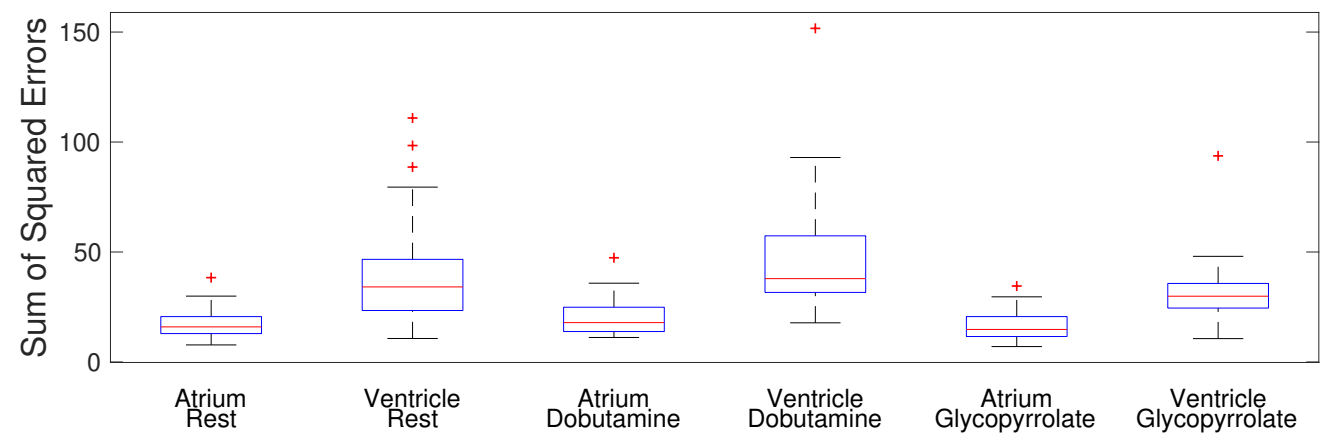

Figure 4: Boxplot for the sum of squared errors between model volume curves for the atrium and ventricle and data during rest, Inotropic stress (Dobutamine), and Chronotropic stress (Glycopyrrolate).

We show in Figures 5, 6, 7 model curves from one elderly woman during rest and pharmacological stress. From Figure 5, we observe the decrease in minimum and maximum volume in the atrium and shorter period of one heart cycle during pharmacological stress. Prominently, we can see the difference during mid diastolic expansion. At rest, the volume is able to increase from mid diastolic expansion and peaks before atrial contraction. This is not present during pharmacological stress. From Figure 6, we also observe the decrease in minimum and maximum volume in the ventricle and shorter period of one heart cycle during pharmacological stress. During pharmacological stress, we see that the volume does not plateau during diastolic filling. From Figure 7, we see the increase in ventricular and arterial pressure during pharmacological stress, suggesting that the heart is exerting more effort in these conditions. Indeed, the model is able to capture blood volume dynamics and is able to give consistent pressure profile. The model curves from optimal parameters for all persons are given in Supplementary B. 

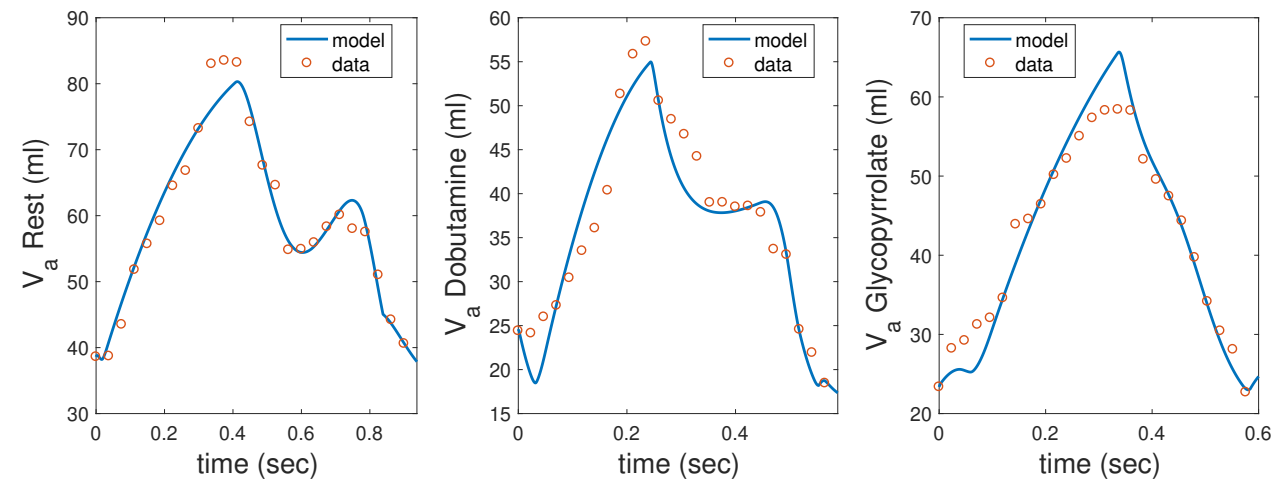

Figure 5: Volume in the atrium for an elderly woman (person 24) during resting condition, dobutamine strees, and glycopyrrolate stress. The SSE between the model curves and data at rest, dobutamine stress, and glycopyrrolate stress are respectively 13.02, 17.17, and 14.02 .
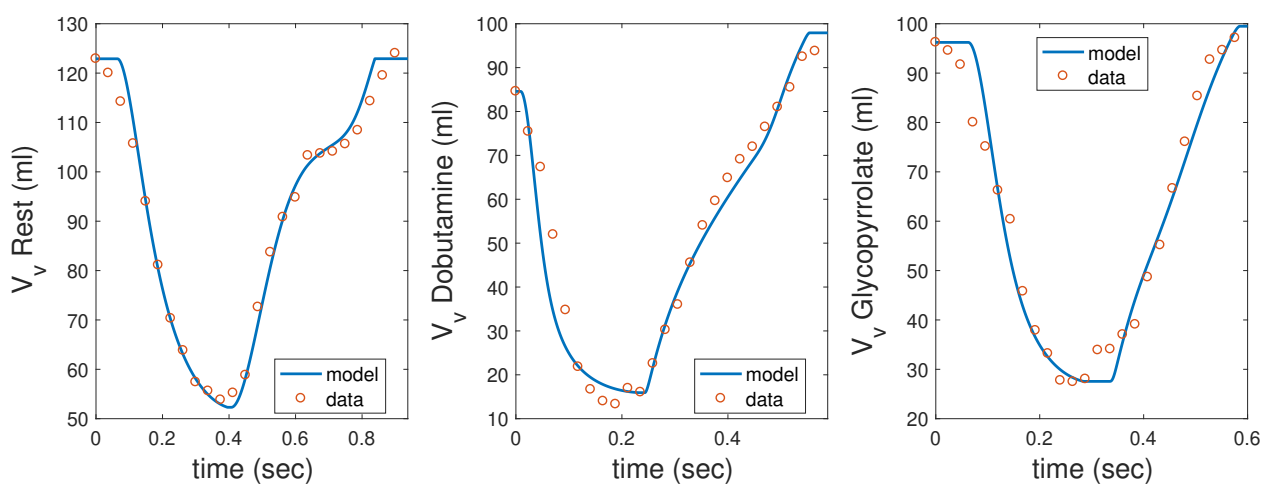

Figure 6: Volume in the ventricle for an elderly woman (person 24) during resting condition, dobutamine strees, and glycopyrrolate stress. The SSE between the model curves and data at rest, dobutamine stress, and glycopyrrolate stress are respectively 13.97, 28.42, and 24.35 . 

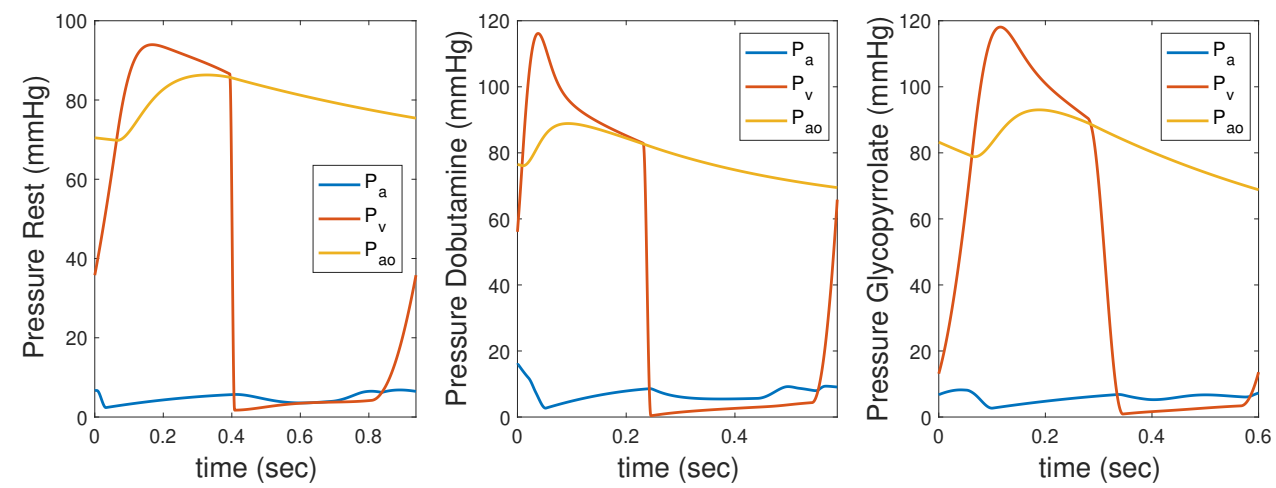

Figure 7: Pressure curves in the different compartments in the model for an elderly woman (person 24) during resting condition, dobutamine strees, and glycopyrrolate stress. The blue, orange, and yellow curves correspond to the pressure in the atrium $P_{a}$, ventricle $P_{v}$, and aorta $P_{a o}$, respectively.

\subsection{Parameter Range of Values}

We collate the different parameter values for each of the 40 persons to obtain a range of values for a given parameter during rest and pharmacological stress. As an illustration, we give in Table 4 the mean \pm standard deviation of the values for maximum ventricular compliance, $C_{v M}$, from all 40 persons and for the subgroups: young vs elderly and women vs men. We present a boxplot visualization for the range of values for $C_{v M}$ in Figure 8. We can observe from the boxplots that there might be a significant difference between $C_{v M}$ of the young vs elderly, and women vs men during rest and pharmacological stress. In general, $C_{v M}$ of the young is higher than the elderly. Also, $C_{v M}$ of the men is higher than the women. The boxplot also suggest that there might be significant differences in even smaller subgroups. For example, we observe from the boxplots that the elderly women have lower $C_{v M}$ when compared to the young women, young men, and elderly 
men. However, due to smaller data sets we do not consider making conclusions regarding these smaller subgroups. A complete boxplot presentation of the range of values for the optimal model parameters for all the persons during rest and pharmacological stress is given in Supplementary C.
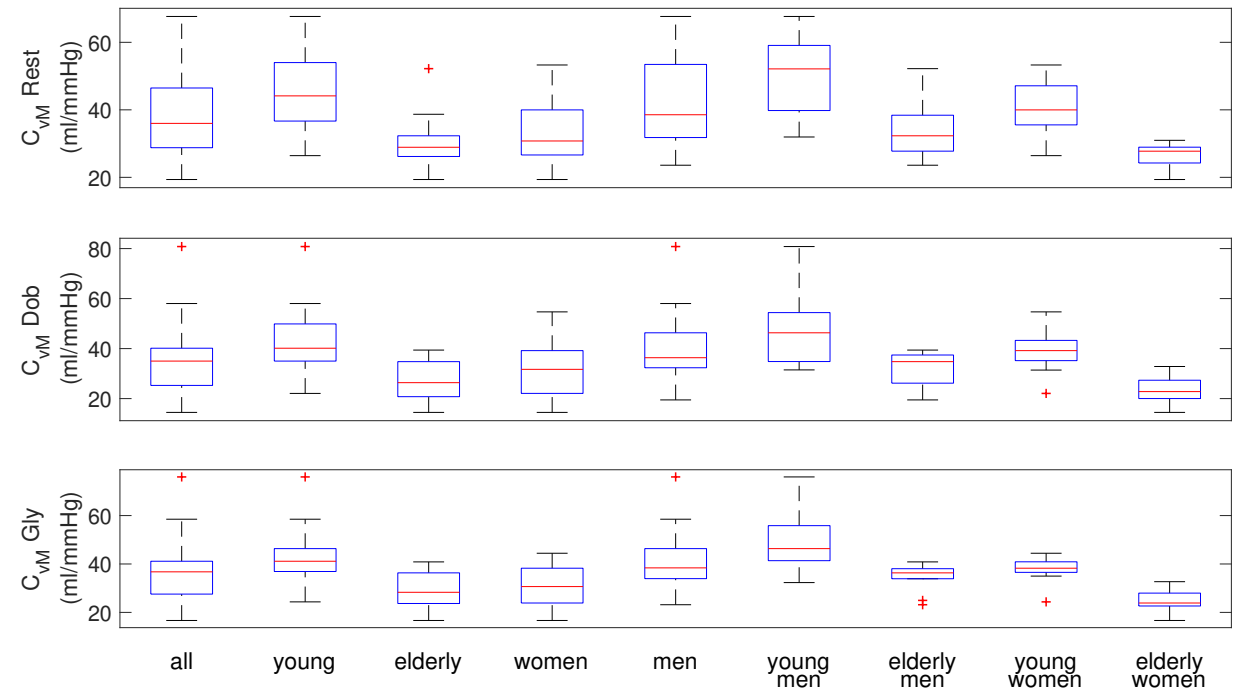

Figure 8: Boxplots for the range of values for the maximum ventricular compliance $C_{v M}$ at resting condition (Rest), dobutamine stress (Dob), and glycopyrrolate stress (Gly) for all 40 persons and different subgroups (young, elderly, women, men, young men, elderly men, young women, and elderly women).

\begin{tabular}{|c|c|c|c|c|c|}
\hline & All & Young & Elderly & Women & Men \\
\hline Rest & $37.86 \pm 11.73$ & $45.30 \pm 10.73$ & $30.42 \pm 7.12$ & $33.63 \pm 9.07$ & $42.08 \pm 12.752$ \\
\hline Dob & $35.20 \pm 12.97$ & $42.96 \pm 12.72$ & $27.44 \pm 7.52$ & $31.02 \pm 10.46$ & $39.37 \pm 14.10$ \\
\hline Gly & $36.40 \pm 11.53$ & $43.16 \pm 11.30$ & $29.63 \pm 7.00$ & $31.26 \pm 8.12$ & $41.53 \pm 12.31$ \\
\hline
\end{tabular}

Table 4: The mean \pm standard deviation of the maximum ventricular compliance for all 40 persons and for the subgroups: Young vs Elderly and Women vs men. 


\subsection{Statistics}

The mean \pm standard deviation for the parameter values for all 40 patients and for subgroups young vs elderly and women vs men during resting condition are shown in Table 5 . The mean \pm standard deviation for the parameter values during pharmacological stress are given in Supplementary D. In this section, we also want to determine whether there are significant differences in mean parameter values between young and elderly, women and men, resting condition and pharmacological stress, and interactions between these factors. To do this, we use the repeated measures model design ANOVA and a statistical significance level of 0.05 . We use the Matlab function fitrm to fit a repeated measures model having 1 within-subjects independent variable and 2 between-subjects independent variable. The within-subjects independent variable is the state of the patients (at rest, during dobutamine stress, and during glycopyrrolate stress). The between-subjects independent variable are age (young and elderly) and sex (women and men). Note that the dependent variable is the value of the model parameter being considered. The repeated measures model is then analyzed using the Matlab function anova to determine between-subjects effects while the Matlab function ranova is used to get within-subject and interaction effects. The anova function performs the analysis of variance for the between-subjects effects where the dependent variable is calculated by taking the average of the within-subjects factor. On the other hand, the ranova function is used to perform the analysis of variance on the repeated measures model (The Mathworks, Inc.). For the repeated measures ANOVA, the mauchly function in Matlab is used to test for sphericity. In the case where sphericity does not hold, the adjusted 
$p$-value calculated through Greenhouse-Geisser approximation is taken when $p<0.75$. Otherwise, we use the Huynh-Feldt approximation (Howell (2002);

Field (2013)).

\begin{tabular}{|c|c|c|c|c|c|c|}
\hline Parameter & Units & All & Young & Elderly & Women & Men \\
\hline$P_{p}$ & $\mathrm{mmHg}$ & $8.27 \pm 2.31$ & $9.18 \pm 2.76$ & $7.35 \pm 1.26$ & $8.15 \pm 3.01$ & $8.38 \pm 1.38$ \\
\hline$b_{a} / 10^{-3}$ & $\mathrm{mmHg} \cdot \mathrm{s}^{2} / \mathrm{ml}^{2}$ & $0.21 \pm 0.17$ & $0.25 \pm 0.23$ & $0.17 \pm 0.07$ & $0.25 \pm 0.23$ & $0.16 \pm 0.08$ \\
\hline$L_{a} / 10^{-4}$ & $\mathrm{mmHg} \cdot \mathrm{s}^{2} / \mathrm{ml}$ & $0.54 \pm 0.19$ & $0.52 \pm 0.17$ & $0.56 \pm 0.21$ & $0.57 \pm 0.20$ & $0.51 \pm 0.18$ \\
\hline$V_{a, u}$ & $\mathrm{ml}$ & $12.69 \pm 3.67$ & $11.74 \pm 3.80$ & $13.63 \pm 3.36$ & $10.99 \pm 3.44$ & $14.38 \pm 3.11$ \\
\hline$C_{a m}$ & $\mathrm{ml} / \mathrm{mmHg}$ & $3.31 \pm 1.82$ & $3.11 \pm 2.20$ & $3.50 \pm 1.36$ & $2.80 \pm 1.15$ & $3.81 \pm 2.22$ \\
\hline$C_{a M}$ & $\mathrm{ml} / \mathrm{mmHg}$ & $11.92 \pm 3.49$ & $10.88 \pm 3.77$ & $12.96 \pm 2.93$ & $10.88 \pm 2.23$ & $12.95 \pm 4.22$ \\
\hline$T_{a s, f r a c}$ & $\%$ of $T$ & $24.27 \pm 6.75$ & $22.74 \pm 8.00$ & $25.80 \pm 4.95$ & $24.57 \pm 7.18$ & $23.97 \pm 6.46$ \\
\hline$T_{a r, f r a c}$ & $\%$ of $T$ & $4.22 \pm 1.61$ & $4.23 \pm 2.09$ & $4.20 \pm 0.99$ & $4.57 \pm 2.06$ & $3.87 \pm 0.92$ \\
\hline$D T_{\text {frac }}$ & $\%$ of $T$ & $15.76 \pm 6.29$ & $13.27 \pm 6.98$ & $18.25 \pm 4.42$ & $14.90 \pm 7.37$ & $16.61 \pm 5.04$ \\
\hline$R_{v m}$ & $\mathrm{mmHg} \cdot \mathrm{s} / \mathrm{ml}$ & $0.0074 \pm 0.0018$ & $0.0072 \pm 0.0018$ & $0.0076 \pm 0.0018$ & $0.0078 \pm 0.0014$ & $0.007 \pm 0.002$ \\
\hline$L_{v} / 10^{-3}$ & $\mathrm{mmHg} \cdot s^{2} / \mathrm{ml}$ & $0.42 \pm 0.15$ & $0.40 \pm 0.15$ & $0.43 \pm 0.15$ & $0.39 \pm 0.14$ & $0.44 \pm 0.16$ \\
\hline$V_{v, u}$ & $\mathrm{ml}$ & $7.93 \pm 1.26$ & $7.98 \pm 1.34$ & $7.88 \pm 1.21$ & $7.09 \pm 1.11$ & $8.76 \pm 0.75$ \\
\hline$C_{v m}$ & $\mathrm{ml} / \mathrm{mmHg}$ & $0.46 \pm 0.14$ & $0.53 \pm 0.11$ & $0.39 \pm 0.13$ & $0.40 \pm 0.12$ & $0.51 \pm 0.15$ \\
\hline$C_{v M}$ & $\mathrm{ml} / \mathrm{mmHg}$ & $37.86 \pm 11.73$ & $45.30 \pm 10.73$ & $30.42 \pm 7.12$ & $33.63 \pm 9.07$ & $42.08 \pm 12.75$ \\
\hline$T_{v s, f r a c}$ & $\%$ of $T$ & $45.93 \pm 6.38$ & $43.57 \pm 6.88$ & $48.30 \pm 4.94$ & $48.43 \pm 5.90$ & $43.44 \pm 5.97$ \\
\hline$T_{v r, f r a c}$ & $\%$ of $T$ & $4.34 \pm 1.53$ & $4.34 \pm 1.43$ & $4.33 \pm 1.66$ & $4.70 \pm 1.73$ & $3.98 \pm 1.25$ \\
\hline$R_{\text {aom }}$ & $\mathrm{mmHg} \cdot s / \mathrm{ml}$ & $0.0308 \pm 0.013$ & $0.0277 \pm 0.013$ & $0.0338 \pm 0.0127$ & $0.0332 \pm 0.0157$ & $0.0283 \pm 0.0094$ \\
\hline$L_{a o} / 10^{-4}$ & $\mathrm{mmHg} \cdot \mathrm{s}^{2} / \mathrm{ml}$ & $0.19 \pm 0.03$ & $0.19 \pm 0.03$ & $0.19 \pm 0.02$ & $0.19 \pm 0.03$ & $0.19 \pm 0.02$ \\
\hline$V_{a o, u}$ & $\mathrm{ml}$ & $310.08 \pm 58.98$ & $306.96 \pm 55.62$ & $313.20 \pm 63.45$ & $264.63 \pm 31.04$ & $355.53 \pm 42.75$ \\
\hline$C_{a o}$ & $\mathrm{ml} / \mathrm{mmHg}$ & $2.89 \pm 0.57$ & $2.77 \pm 0.61$ & $3.01 \pm 0.51$ & $2.53 \pm 0.47$ & $3.24 \pm 0.42$ \\
\hline$R_{p f}$ & $\mathrm{mmHg} \cdot \mathrm{s} / \mathrm{ml}$ & $0.26 \pm 0.08$ & $0.27 \pm 0.07$ & $0.26 \pm 0.08$ & $0.30 \pm 0.08$ & $0.23 \pm 0.05$ \\
\hline$P_{p f}$ & $\mathrm{mmHg}$ & $57.97 \pm 6.27$ & $58.68 \pm 7.25$ & $57.25 \pm 5.19$ & $57.13 \pm 4.00$ & $58.81 \pm 7.95$ \\
\hline
\end{tabular}

Table 5: Mean \pm standard deviation for all 40 persons during resting condition and for subgroups: Young vs Elderly and Women vs Men. For similar table under pharmacological stress see Supplementary D.

Young and Elderly. We found that there was a significant effect of age on the parameter values of $P_{p}, V_{a, u}, C_{a M}, R_{v m}, C_{v m}, C_{v M}, T_{v s, f r a c}, R_{a o m}$, 
and $P_{p f}$. Strictly speaking, it means the hypothesis that these average value across the within-subjects model are equal is rejected, which is commonly interpreted as these parameters are significantly different between young vs elderly. The young have lower marginal mean for $V_{a, u}(9.55 \pm 3.81$ vs $11.86 \pm$ $3.34 \mathrm{ml}, p=0.001), C_{a M}(9.75 \pm 3.17 \mathrm{vs} 11.84 \pm 3.08 \mathrm{ml} / \mathrm{mmHg}, p=0.015)$, $R_{v m}(0.0069 \pm 0.0018$ vs $0.0084 \pm 0.0028 \mathrm{mmHg} \cdot \mathrm{s} / \mathrm{ml}, p=0.005), T_{v s, \text { frac }}$ $(45.59 \pm 7.34$ vs $51.24 \pm 6.87 \%$ of $T, p<0.001)$, and $R_{\text {aom }}(0.021 \pm 0.09$ vs $0.22 \pm 0.08 \mathrm{mmHg} \cdot \mathrm{s} / \mathrm{ml}, p=0.0495)$. On the other hand, the young have higher marginal mean for $P_{p}(8.94 \pm 1.86$ vs $7.53 \pm 1.19 \mathrm{mmHg}, p<0.001)$, $C_{v m}(0.50 \pm 0.18$ vs $0.29 \pm 0.16 \mathrm{ml} / \mathrm{mmHg}, p<0.001), C_{v M}(43.81 \pm$ 11.46 vs $29.16 \pm 7.21 \mathrm{ml} / \mathrm{mmHg}, p<0.001), R_{p f}(0.24 \pm 0.09$ vs 0.22 $\pm 0.08 \mathrm{mmHg} \cdot \mathrm{s} / \mathrm{ml}, p=0.008)$, and $P_{p f}(60.85 \pm 10.39$ vs $56.82 \pm 7.15$ $\mathrm{mmHg}, p=0.003)$. A visualization of the significantly different parameters between the young and elderly is given in Figure 9. The marginal means of all parameters for the young vs elderly are given in Supplementary D. 


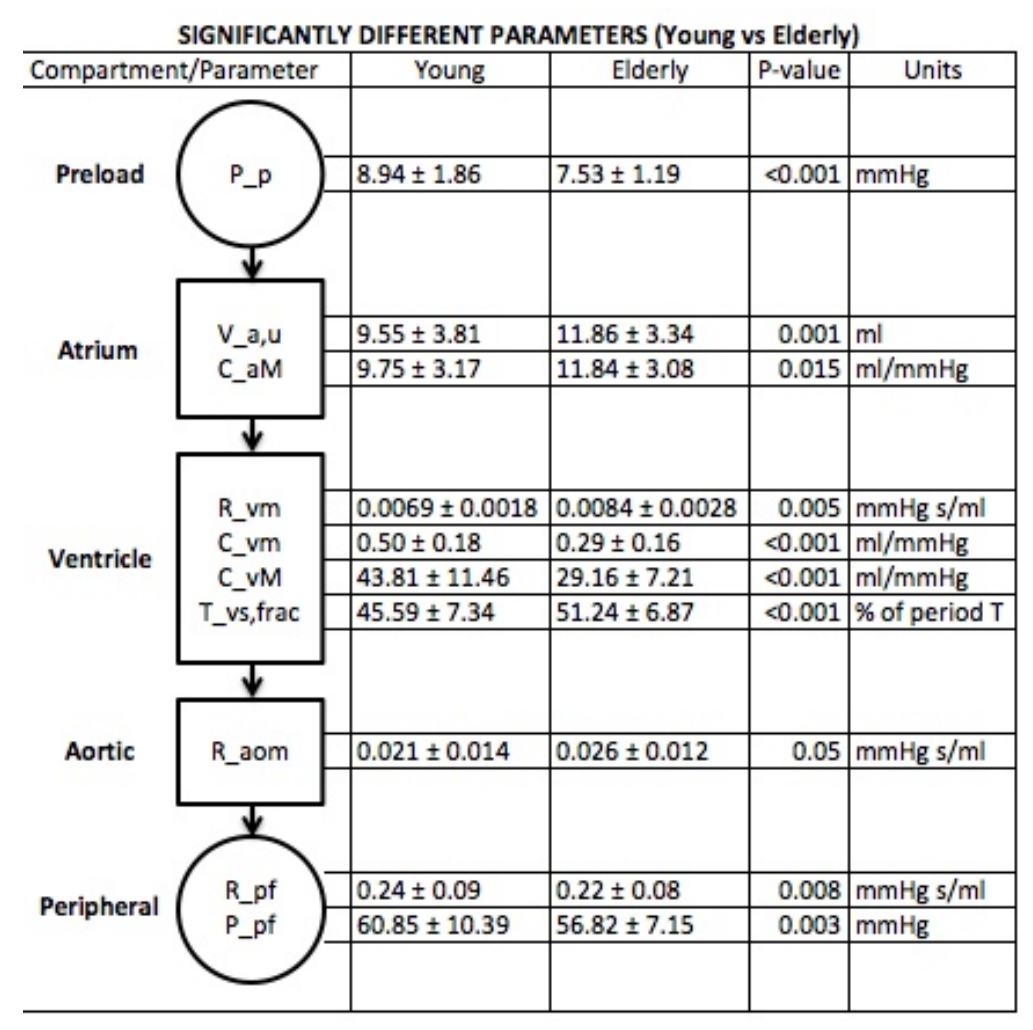

Figure 9: Model parameters that are significantly different between the young vs elderly when the parameter values are averaged across resting condition, dobutamine stress, and glycopyrrolate stress.

Women and Men. We found that there was a significant effect of sex on the parameter values of $b_{a}, V_{a, u}, C_{a M}, R_{v m}, V_{v, u}, C_{v m}, C_{v M}, T_{v s, f r a c}, R_{a o m}$, $V_{a o u}, C_{a o}, R_{p f}$, and $P_{p f}$. Strictly speaking, it means the hypothesis that these average value across the within-subjects model are equal is rejected, which is commonly interpreted as these parameters are significantly different between women vs men. The women have lower marginal mean for $V_{a, u}(8.83$ \pm 3.35 vs $12.57 \pm 3.17 \mathrm{ml}, p<0.001), C_{a M}(9.83 \pm 2.65$ vs $11.76 \pm 3.59$ 
$\mathrm{ml} / \mathrm{mmHg}, p=0.024), V_{v, u}(7.25 \pm 1.86$ vs $9.23 \pm 1.95 \mathrm{ml}, p<0.001)$, $C_{v m}(0.35 \pm 0.18$ vs $0.44 \pm 0.21 \mathrm{ml} / \mathrm{mmHg}, p=0.009), C_{v M}(31.97 \pm 9.19$ vs $41.00 \pm 12.91 \mathrm{ml} / \mathrm{mmHg}, p<0.001), V_{a o, u}(235.94 \pm 61.76$ vs $317.26 \pm$ $91.73 \mathrm{ml}, p<0.001), C_{a o}(3.02 \pm 0.91$ vs $3.72 \pm 1.07 \mathrm{ml} / \mathrm{mmHg}, p<0.001)$, and $P_{p f}(56.04 \pm 7.34$ vs $61.63 \pm 9.88 \mathrm{mmHg}, p<0.001)$. On the other hand, the women have higher marginal mean for $b_{a}(0.15 \pm 0.15$ vs $0.11 \pm$ $\left.0.06 \mathrm{mmHg} \cdot \mathrm{s}^{2} / \mathrm{ml}^{2}, p=0.022\right), R_{v m}(0.0083 \pm 0.0024$ vs $0.007 \pm 0.0024$ $\mathrm{mmHg} \cdot \mathrm{s} / \mathrm{ml}, p=0.012), T_{v s, f r a c}(50.98 \pm 7.59$ vs $45.85 \pm 6.81 \%$ of the period $T, p<0.001), R_{\text {aom }}(0.026 \pm 0.016 \mathrm{vs} 0.021 \pm 0.009 \mathrm{mmHg} \cdot \mathrm{s} / \mathrm{ml}$, $p=0.023)$, and $R_{p f}(0.24 \pm 0.09$ vs $0.22 \pm 0.08 \mathrm{mmHg} \cdot \mathrm{s} / \mathrm{ml}, p=0.008)$. A visualization of the significantly different parameters between the women and men is given in Figure 10. The marginal means of all parameters for the women vs men are given in Supplementary D. 


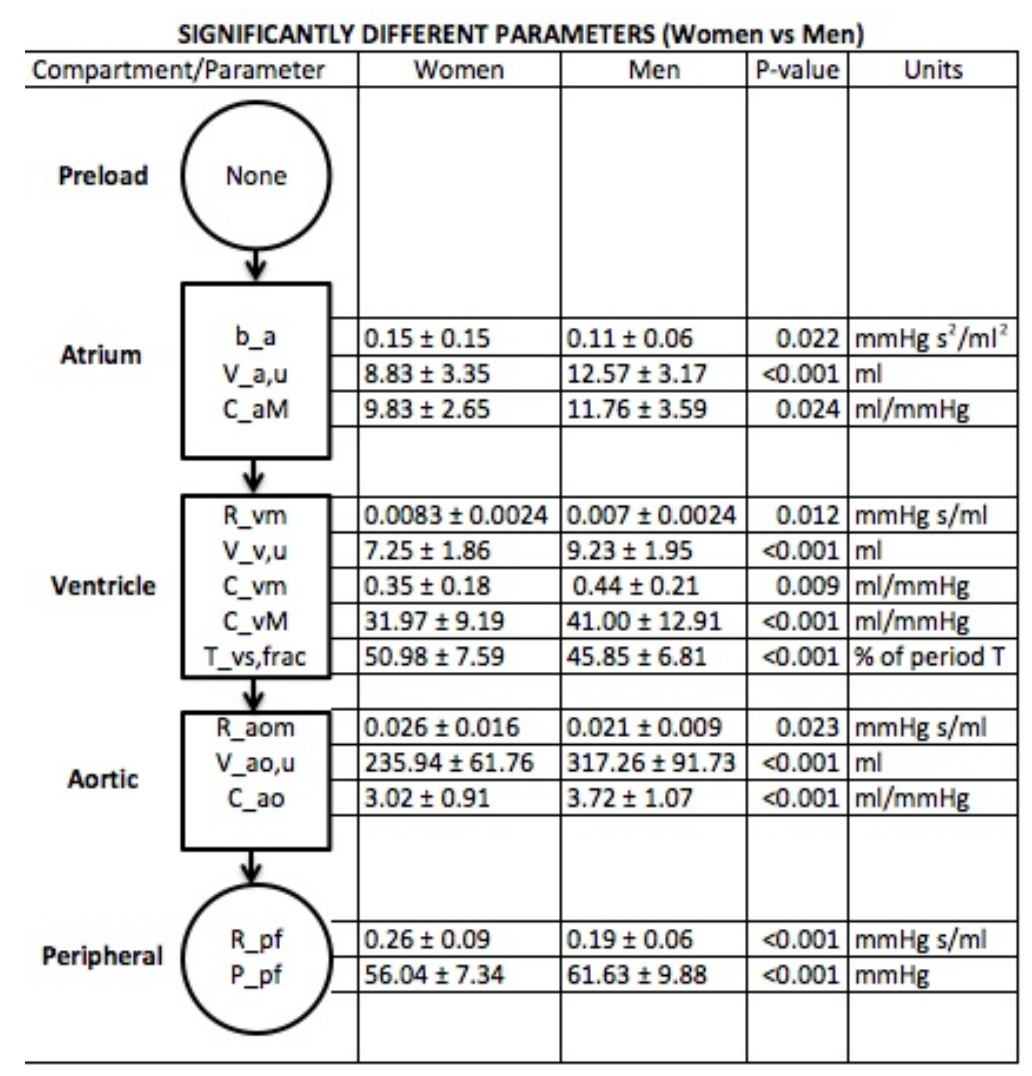

Figure 10: Model parameters that are significantly different between women vs men when the parameter values are averaged across resting condition, dobutamine stress, and glycopyrrolate stress.

Effects of Dobutamine and Glycopyrrolate. We found that there was a significant effect of state of the patients on the parameter values of $b_{a}$, $L_{a}, V_{a, u}, C_{a m}, C_{a M}, T_{a s, f r a c}, T_{a r, f r a c}, D T_{f r a c}, R_{v m}, L_{v}, C_{v m}, T_{v s, f r a c}, T_{v r, f r a c}$, $R_{a o m}, L_{a o}, V_{a o, u}, C_{a o}, R_{p f}$, and $P_{p f}$. Indicating the hypothesis that the mean values of these parameters without considering between subjects effect for any state are all equal is rejected. This is commonly interpreted as the mean values of these parameters for at least two states are significantly different. 
To determine which states are significantly different for these parameters, we perform post hoc test using the Matlab function multcompare. Multcompare is able to perform multiple pairwise comparison of treatment effects or group means with different options to compensate for multiple tests. We choose the default option Tukey's honesty significance difference criterion. In particular we are interested on the parameters that have significantly different mean values during rest vs dobutamine stress and during rest vs glycopyrrolate stress. We have found that the mean values of the parameters $b_{a}, L_{a}, V_{a, u}$, $C_{a m}, C_{a M}, T_{a s, f r a c}, D T_{f r a c}, L_{v}, C_{v m}, R_{a o m}, L_{a o}, V_{a o, u}, C_{a o}$, and $R_{p f}$ are significantly different at rest vs during dobutamine stress. Furthermore, the mean values of the parameters $b_{a}, L_{a}, V_{a, u}, C_{a M}, T_{a s, f r a c}, T_{a r, f r a c}, D T_{f r a c}$, $L_{v}, T_{v s, f r a c}, T_{v r, f r a c}, R_{a o m}, L_{a o}$, and $V_{a o, u}$ are significantly different at rest vs during glycopyrrolate stress. Figure 11 gives the percentage increase or decrease of parameters having significant change from rest vs dobutamine stress and rest vs glycopyrrolate stress. 


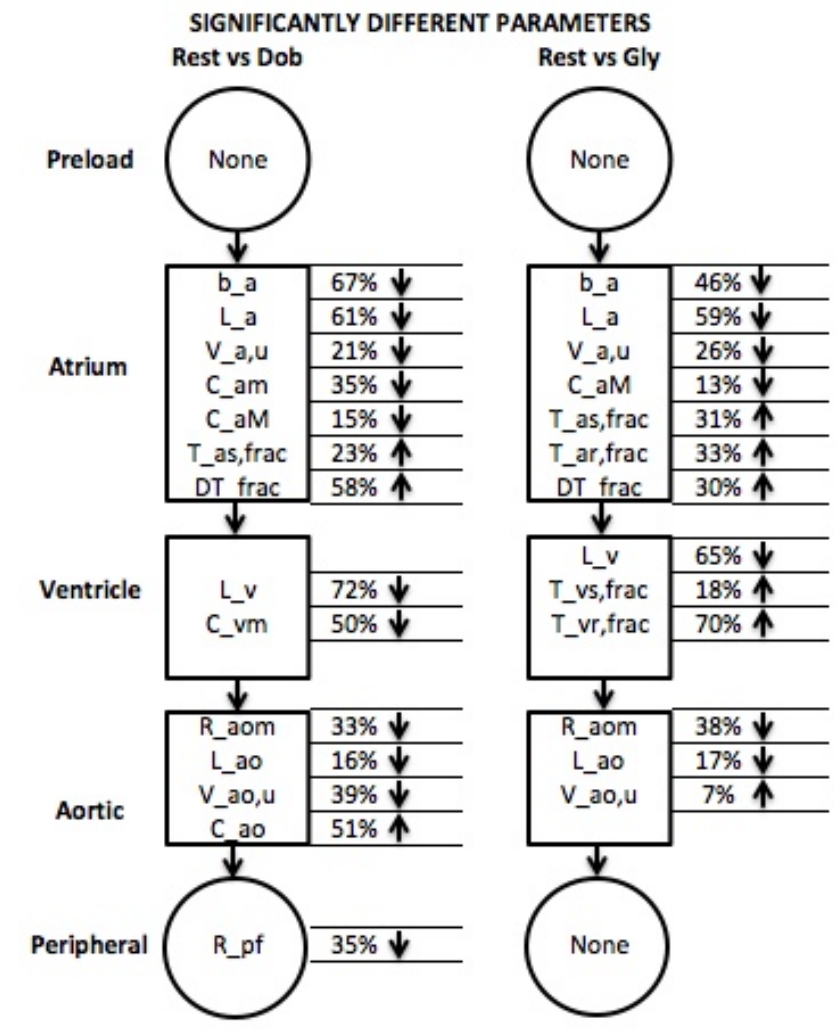

Figure 11: The percentage increase or decrease of parameters having significant change from rest vs dobutamine (Dob) stress and rest vs glycopyrrolate (Gly) stress. An arrow pointing upward is used to indicate an increase while an arrow pointing downward is for a decrease.

Interaction effects. We have found significant interaction for age and sex only for $L_{a}$, age and state of patients for $V_{a u}, V_{v u}, C_{v m}$, and $T_{v r, f r a c}$, sex and state of patients for $C_{v m}, V_{a o u}$, and $C_{a o}$, and none for age, sex, and state of patients. 


\section{Discussion}

In this study, we have established that our model can be calibrated well to data during resting conditions and inotropic (dobutamine) and chronotropic (glycopyrrolate) pharmacological stress. We have shown that a simple cardiovascular model involving the atrium and ventricle can capture the dynamics of blood flow in the left heart. Medical doctors believe that some indications of heart diseases can be seen or spotted earlier through evaluation of atrial performance (Douglas (2003); Aurigemma et al. (2009); Vieira et al. (2014)). Thus, the addition of the compartment for the atrium that is able to capture realistic behavior is a helpful step forward in cardiovascular modeling. It is also informative in understanding hardly assessible parts of the cardiovascular system in general. Through the model we were able to get a visualization of the range of values of different model parameters. These ranges of model parameter values translate to understanding the physiological concepts they represent. Also, a statistical test comparing the mean values of a given parameter for the subgroups young vs elderly and women vs men gave us a way to visualize differences between the young and the elderly and also between women and men. Finally, we were able to quantify the effects of the drugs dobutamine and glycopyrrolate on different heart functions.

\subsection{Model Development}

In the compartmental model of blood circulation given in Olufsen et al. (2005), most of the system differential equations are in terms of change in pressure, $d P / d t$. They were obtained from differentiation of the linear rela- 
tion of volume and pressure, $V=c P$. This gives the equation

$$
\frac{d V}{d t}=c \frac{d P}{d t}+P \frac{d c}{d t}=Q_{\text {in }}-Q_{\text {out }}
$$

Through the utilization of volume in the compartments as state variables in our model, we were able to do away with taking the rate of change of the time dependent compliance. Also, we can directly fit the model volume curves with data and allows patient specific data on volumes to be used as initial values of the state variables. The use of conductance instead of resistance gives a way of representing a non-leaking heart valve without using an infinite number. Note that a conductance having value zero is equivalent to an infinitely large resistance. In addition, we have less parameters to identify. On the use of the unsteady Bernoulli effect to represent flow into the atrium, $Q_{a}$, we recall from Section 2.2.3 that this makes us do away with the ad-hoc formulation of the resistance to flow into the atrium. Furthermore, we are able to have one less parameter to optimize. Also, the use of the unsteady Bernoulli effect displayed that the optimization is able to improve the fit between model volume curves and volume data in the atrium and ventricle (see Figure 12). Using the unsteady Bernoulli effect, the mean SSE between model volume curves for all persons and data in the atrium and ventricle are $17.43 \pm 6.23$ and $38.61 \pm 22.42$, respectively. These are improvements of the mean SSE between the model volume curves from the Open Differential model and data, which are $22.36 \pm 12.05$ and $46.27 \pm 35.59$ for the atrium and ventricle, respectively. 

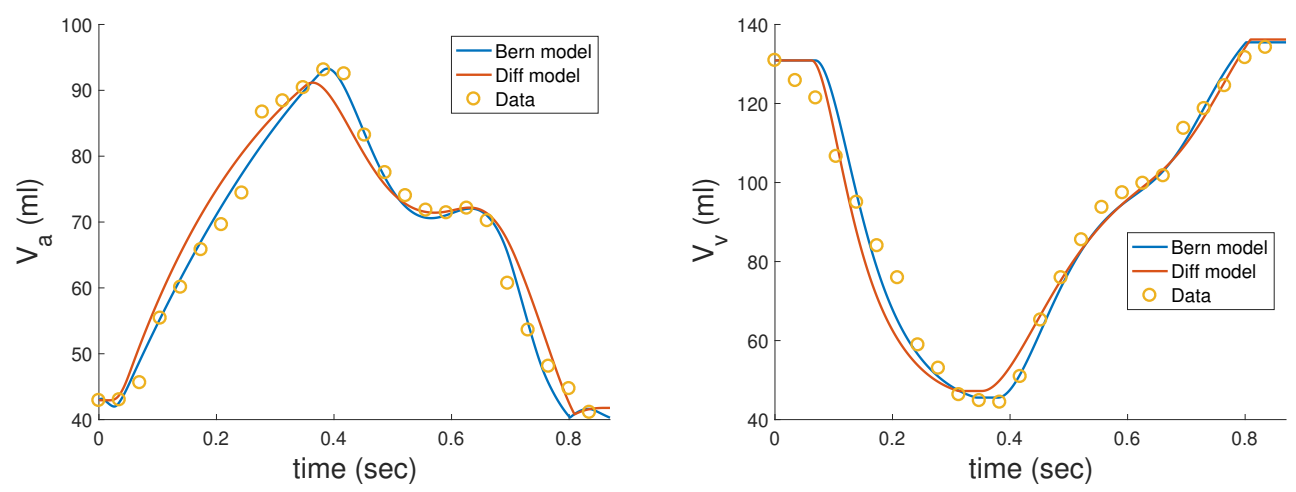

Figure 12: Model volume curves at rest for an elderly female (person 22) from the Open Differential Model, abbreviated as Diff model (orange curve) and Open Bernoulli-Differential Model, abbreviated as Bern model (blue curve). (Left Panel:) Atrial Volume (SSE between Bern model and data is 10.78 and SSE between Diff model and data is 18.59) (Right Panel:) Ventricular volume (SSE between Bern model and data is 22.83 and SSE between Diff model and data is 27.92)

\subsection{Bootstrapping}

Noise in the data can be a major source of uncertainty for parameter estimates for a given model. We use a bootstrapping method, which is described comprehensively in Chowell (2017), to quantify parameter uncertainty that may be caused by possible noise in the data. In outline, pseudo datasets on blood volume in the atrium and ventricle are generated from Log Normal Distributions with mean that gives the best fit to the experimental data and standard deviation set to $5 \mathrm{ml}$. The best fit curves are the blood volume curves corresponding to the optimal parameters. We assume a Log Normal Distribution for the noise to get nonnegative and continuous pseudo blood volume data. Next, optimal parameters are estimated from each of pseudo datasets to generate a new set of optimal parameters. The collection of all 
the optimal parameters corresponding to each of the pseudo datasets are then used to characterize parameter uncertainty and construct confidence intervals about their means. We perform the above bootstrapping method for all subjects at resting condition and during pharmacological stress. For most of the subjects, we were able to get the same set of optimal parameters from 1000 bootstrap realizations. We observe that the length of the confidence interval for the sample mean of the parameters are small. In particular, most of them have length zero. Hence, we can say that that the model parameters are reliable in the sense that small noise in the data sets does not cause a significant change in the optimal parameter values. A colormap to visualize the $95 \%$ confidence interval about the mean of the optimal parameters for all patients during rest and pharmacological stress is given in Supplementary G. From the uncertainty of the parameter estimates, we illustrate in Figure 13 a $95 \%$ confidence band around the best fit volume curves in the atrium and ventricle for a young woman.

Another source of parameter uncertainty is the implementation of the local optimization scheme described in section 2.3. To get a picture of this uncertainty, we generate 1000 random points from a uniform distribution in a neighborhood of our a priori parameter estimates. The radius of the neighborhood was set to $10 \%$ of the a priori estimate values. We then perform the optimization scheme using each of these 1000 points as initial parameter guess to the minimization problem given in section 2.3.2. The collection of all the optimal parameters corresponding to each of the initial parameter guesses is then used to characterize parameter uncertainty with respect to perturbation of initial estimates. We perform the said process for an elderly 
male and present in Figure 14 a 95\% confidence band around the best fit volume curves in the atrium and ventricle. The length of the confidence interval for the sample mean of the parameters were found to be small. A colormap corresponding to the length of the confidence interval about the optimal parameters for this subject is given in Supplementary G.
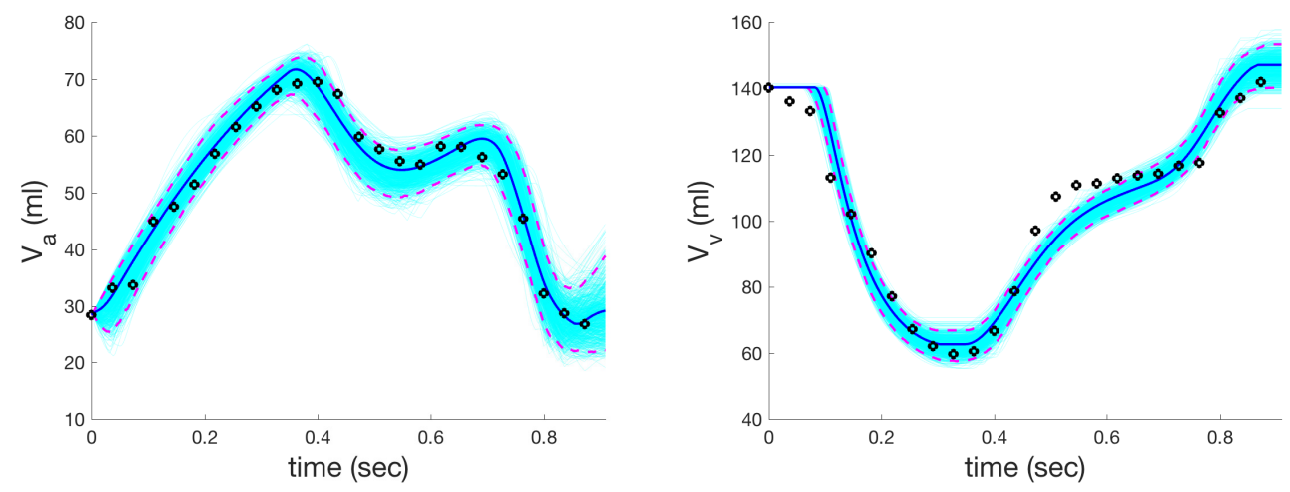

Figure 13: (Left Panel:) Blood volume in the atrium. (Right Panel:) Blood volume in the ventricle. The black circles are blood volume data for a young woman while the blue curves correspond to the best fit of the model to the data, that is, the curves from optimal parameters. The cyan curves correspond to 1000 realizations of the blood volume curves assuming a Log Normal error structure. The dashed magenta lines are $95 \%$ confidence bands around the best fit volume curves in the atrium and ventricle. 

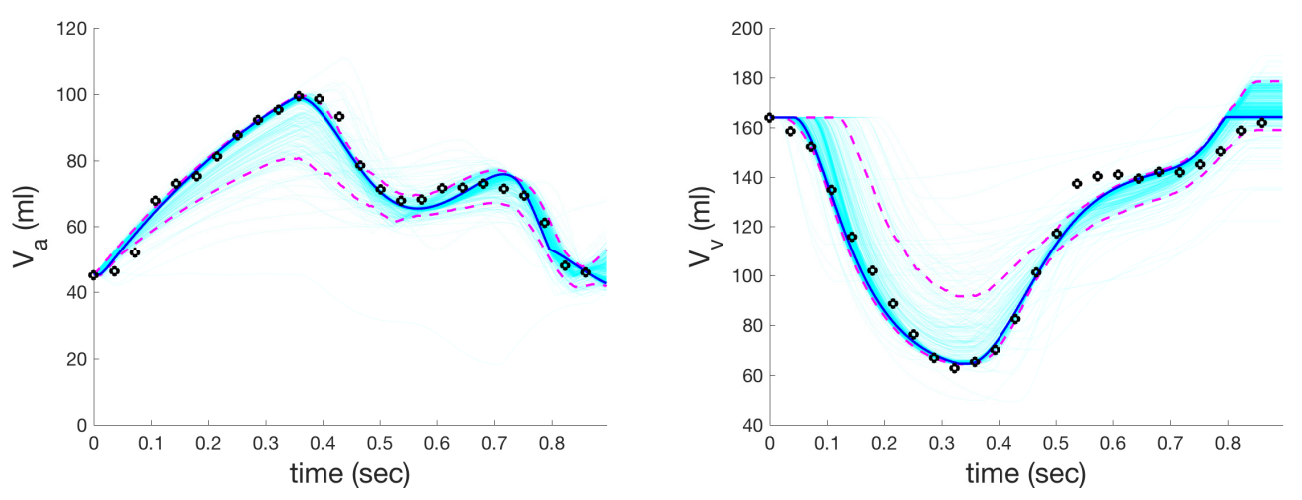

Figure 14: (Left Panel:) Blood volume in the atrium. (Right Panel:) Blood volume in the ventricle. The black circles are blood volume data for an elderly male (person 40) while the blue curves correspond to the best fit of the model to the data, that is, the curves from optimal parameters. The cyan curves correspond blood volume curves from 1000 perturbations of the a priori parameters. The dashed magenta lines are $95 \%$ confidence bands around the best fit volume curves in the atrium and ventricle.

\subsection{Insights into differences in heart function}

For models with patient-specific parameter values, the identification of potential "biomarkers" can be achieved through determining significantly different parameter values between groups (Ottesen (2011a)). This ability of patient-specific modeling is illustrated by the statistical results we have in section 3.3. In addition, we can also use the significant differences between parameter values of different groups as an additional validation of our model. For example, our results show that the $C_{v m}$ and $C_{v M}$ are significantly higher for the young compared to the elderly group. This is in conjunction with the findings that left ventricular compliance was observed to decrease with ageing (Arbab-Zadeh et al. (2004); Fujimoto et al. (2012)). Also, our results show that $C_{v m}, C_{v M}$, and $C_{a o}$ are significantly higher in men compared to 
women. In terms of elastance, this means that women have higher elastance in the ventricle and aortic compartment. Again, this result is in conjunction with the findings that women are more likely to have higher ventricular and aortic compartment elastance through the course of life (Najjar et al. (2004); Redfield et al. (2005)). Also, healthy women showed higher ventricular elastance and diastolic stiffness compared to men (Hayward et al. (2001); Lane et al. (2013)). Indeed, statistical analysis of the patient-specific parameter values between groups can be used to give insights into differences in heart function among these groups and also for supplementary model validation.

\subsection{Effects of Drugs}

Dobutamine. Inotropic stress was introduced through intravenous infusion of dobutamine (Dobutrex). Inotropic effect represents regulation of the heart's power to pump blood or contractility. One such measure of cardiac contractility is maximum ventricular elastance (Suga and Sagawa (1973); Danielsen (1998)). We were able to show that dobutamine caused a significant decrease in minimum ventricular compliance, $C_{v m}$. This means that the drug gave rise to an increase in maximum ventricular elastance or a positive inotropic effect. In addition, we observed that there was a significant decrease in $b_{a}, L_{a}, V_{a, u}, C_{a m}, C_{a M}, L_{v}, R_{a o m}, L_{a o}, V_{a o, u}$, and $R_{p f}$ and an

increase in $T_{a s, f r a c}, D T_{f r a c}$, and $C_{a o}$. Using the total sensitivity defined in Supplementary F (Sensitivity Analysis), the effects of the dobutamine can be ranked according to the effect of the change in parameter values on the model volume curves. In this case, we observe the effect of varying the average parameter values at rest by the percentage changes due to dobutamine on the model volume curves. In particular, we take the sum of the relative 
change in area between the volume curves (atrium, ventricle, and aorta) during rest and during variation due to dobutamine. In this way, we are able to rank which change in parameter value due to dobutamine has the most to least effect on the model volume curves. The effects of dobutamine on the model volume curves and ranking is given in Figure 15 (Top Panel). Indeed, we see that the main effect of dobutamine is a decrease in $C_{v m}$ or an increase in contractility. We note that the Electronic Medicines Compendium (https://www.medicines.org.uk/emc/product/6270) states that dobutamine brings about a positive inotropic effect on the myocardium, facilitates atrioventricular conduction, and may decrease pulmonary vascular resistance.

Glycopyrrolate. Chronotropic stress was introduced through intravenous bolus injection of glycopyrrolate (Robinul). Chronotropic effect represents the increase or decrease of the heart rate. We have observed that glycopyrrolate caused a significant decrease in $b_{a}, L_{a}, V_{a, u}, C_{a M}, L_{v}, R_{a o m}$, and $L_{a o}$ and an increase in $T_{a s, f r a c}, T_{a r, f r a c}, D T_{f r a c}, T_{v s, f r a c}, T_{v r, f r a c}$, and $V_{a o, u}$. As in the case of dobutamine, the effects of glycopyrrolate can be ranked according to the effect of the change in parameter values on the model volume curves. In this case, we observe the effect of varying the average parameter values at rest by the percentage changes due to glycopyrrolate on the model volume curves. In particular, we take the sum of the relative change in area between the volume curves (atrium, ventricle, and aorta) during rest and during variation due to glycopyrrolate. In this way, we are able to rank which change in parameter value due to glycopyrrolate has the most to least effect on the model volume curves. The effects of glycopyrrolate on the model volume curves and ranking is given in Figure 15 (Bottom Panel). We see that 
the main effect of glycopyrrolate is an increase in $T_{v s, f r a c}$ or an increase in contraction time of the ventricle relative to the period of one heart cycle.
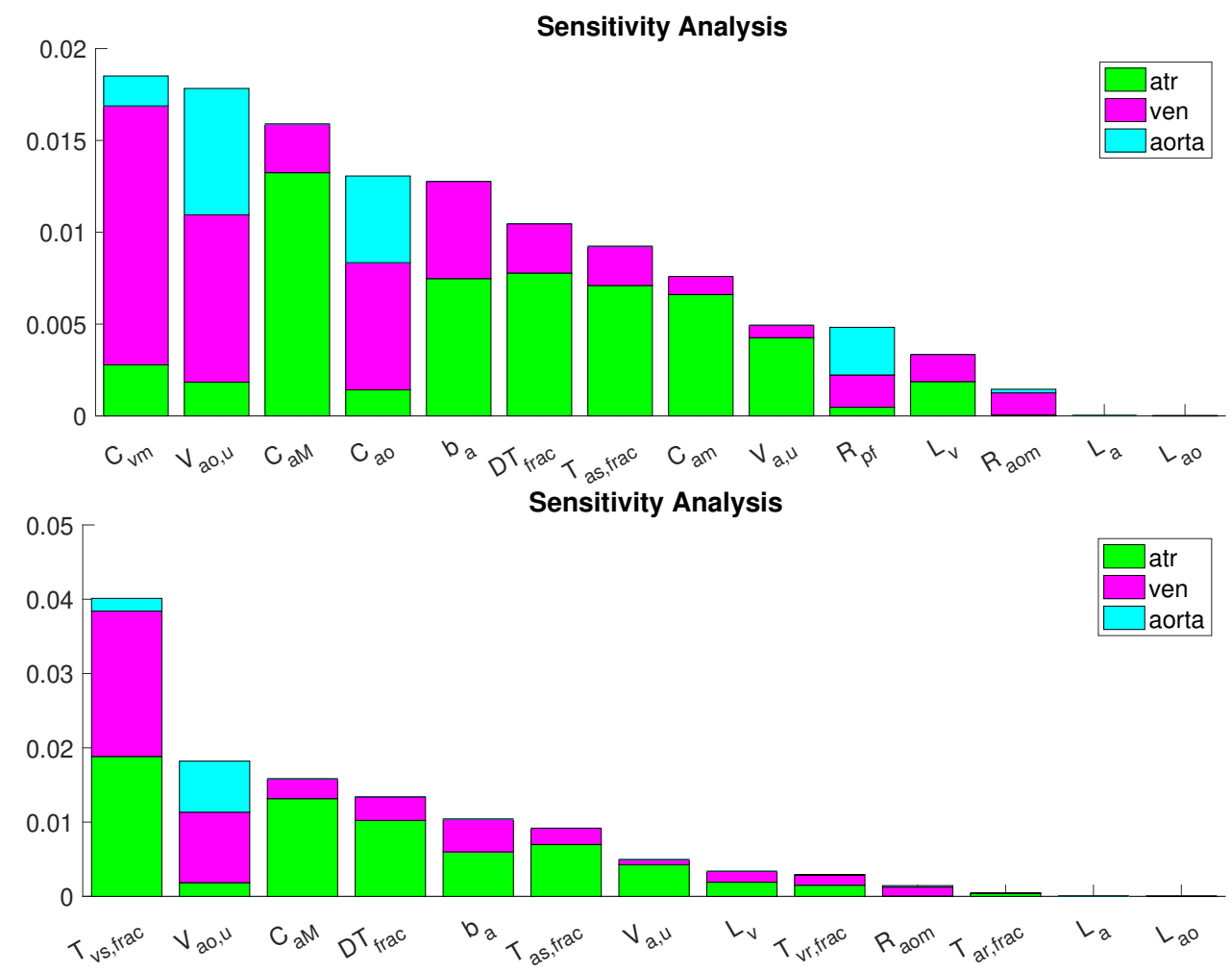

Figure 15: The relative change in area between the volume curves of the atrium (green), ventricle (magenta), and aorta (cyan) during rest and during variation of a parameter due to dobutamine (Top Panel) and glycopyrrolate (Bottom Panel). The change in parameter values due to the drugs are ranked according to their total effect on the model volume curves.

\subsection{Leaking Heart Valve Inspection}

The prevalence of heart valve disease like aortic stenosis and mitral regurgitation becomes greater through age. These diseases are usually treated surgically but it is risky especially for elderly patients (Rostagno (2019)). 
In relation to this, we want to use the model to inspect how a leaky heart valve affects volume curves in the atrium, ventricle, and arteries. We hope that indications in these curves can give insight for early diagnosis and thus maybe lead to doing away with surgery in the longterm. To do this, we use the average of the optimal model parameter values from the elderly group but with a finite value for the maximum resistance to flow into the ventricle for mitral regurgitation and a finite value for the maximum resistance to flow into the aorta for aortic stenosis.

To examine mitral regurgitation, we look at test simulations where $R_{v M}$ is set at 10,5 , and $1 \mathrm{mmHg} \cdot \mathrm{s} / \mathrm{ml}$. Respectively, these correspond to approximately $3.31,6.57$, and $30.85 \mathrm{ml}$ regurgitating from the ventricle to the atrium in one heart cycle. The model curves corresponding to these cases are shown in Figure 16. We see that a leaky mitral valve translates to an increase in peak volume in the atrium, a decrease in minimum volume and increase in mid to end diastolic volume in the ventricle, a decrease in volume in the arteries, and a faster flow into the ventricle at the start of diastolic filling. We observe that the effect of mitral regurgitation on blood volumes is most prominent in the atrium. 

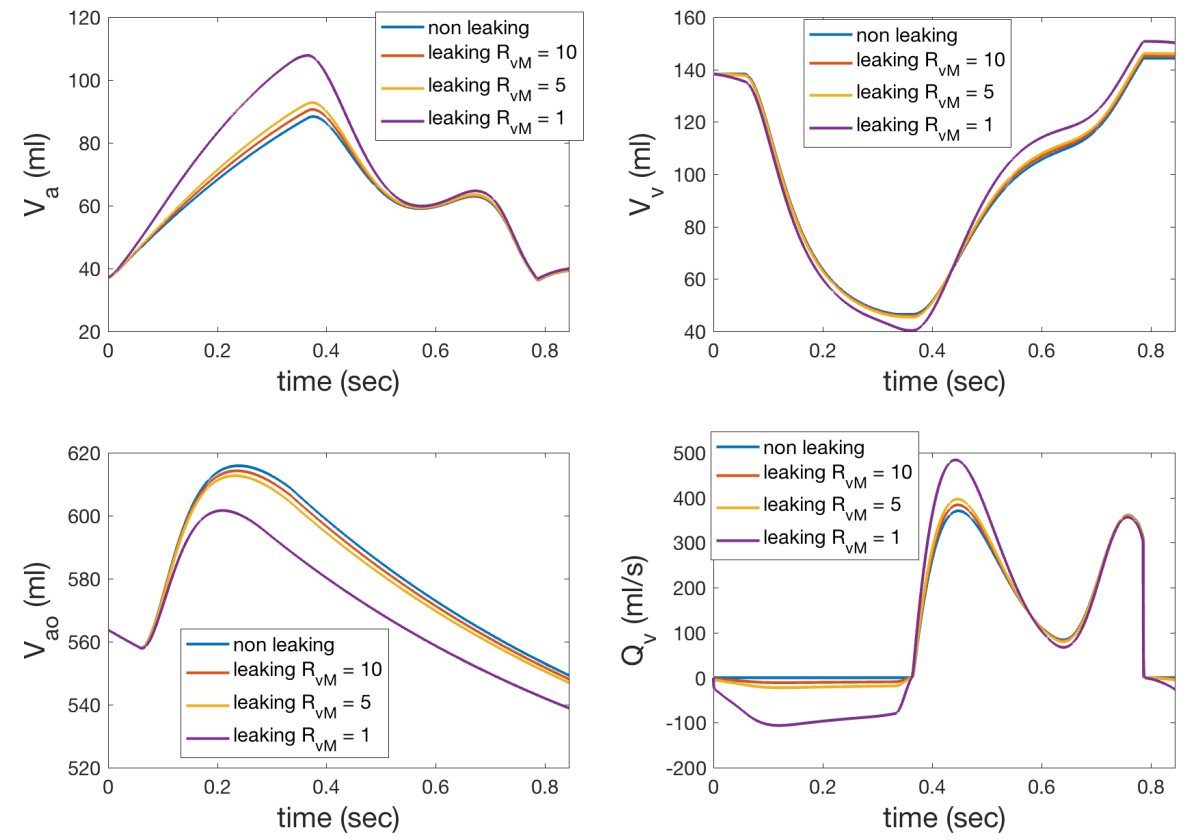

Figure 16: Simulation of a leaky mitral valve by decreasing the maximum resistance to flow into the ventricle from infinity, to 10,5 , and then $1 \mathrm{mmHg} \cdot \mathrm{s} / \mathrm{ml}$. These correspond respectively to a regurgitating volume of approximately $0,3.31,6.57$, and $30.85 \mathrm{ml}$ back into the atrium.

To examine aortic stenosis, we simulate model curves corresponding to a finite value of 10,5 , and $1 \mathrm{mmHg} \cdot \mathrm{s} / \mathrm{ml}$ for maximum aortic resistance. These values correspond to approximately $4.20,8.33$, and $39.14 \mathrm{ml}$ regurgitating from the aorta to the ventricle in one heart beat, respectively. The model curves corresponding to these cases are shown in Figure 17. We observe that a leaky aortic valve translates to an increase in atrial volume during mid diastolic expansion, an increase in diastolic filling volume in the ventricle, and a decrease in arterial volume during the diastolic phase of the ventricle. 
We observe that the effects of aortic regurgitation on the blood volumes can be noticed in the atrium.
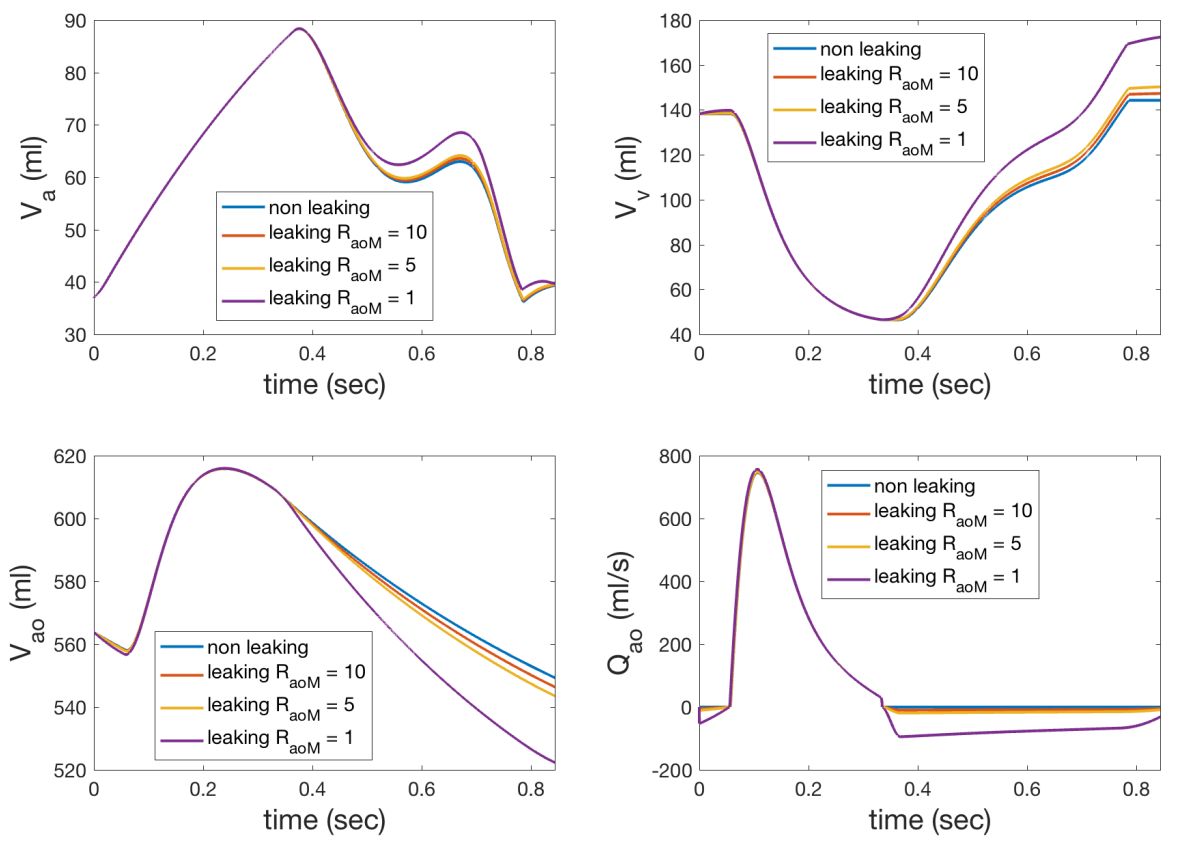

Figure 17: Simulation of a leaky aortic valve by decreasing the maximum resistance to flow into the aorta from infinity, to 10,5 , and then $1 \mathrm{mmHg} \cdot \mathrm{s} / \mathrm{ml}$. These correspond respectively to a regurgitating volume of approximately $0,4.20,8.33$, and $39.14 \mathrm{ml}$ back into the ventricle.

\subsection{Limitations.}

In this study, the model parameters related to the optimal fit were obtained through numerical methods that require important consideration. First, the acquired optimal parameters represents a local solution to the optimization problem. The parameters associated with the local solution 
may not be guaranteed to be within the physiological range. Also, it may be possible to have different sets of parameters that define similar model states (Pope et al. (2011)). Second, the model output are insensitive to some of the model parameters. Thus again, the estimates may not be feasible in a physiological perspective (Kelley (1999)). Also, there might be high correlation between model parameters. We address this considerations by using initial parameter values that are derived from patient-specific data and related studies. Thus in a way guaranteeing that our local solution is near the true solution. Also, the model has been calibrated well with 40 persons under resting conditions, inotropic stress, and chronotropic stress. Furthermore, parametric bootstrapping was performed to establish reliability of the parameter estimates. Lastly, statistical analysis between the obtained mean values of the parameters agree with physiological findings thus again supporting the idea that the optimal parameters may be within physiological range.

\section{Summary}

In conclusion, we have developed a simple compartmental heart model involving the atrium, ventricle, veins, and arteries that can be validated with experimental results. Our model illustrated the use of volume in the compartments as state variables, the use of conductance instead of resistance to represent perfectly closing heart valves, and the use of the unsteady Bernoulli equation to represent blood flow into the atrium. We have shown that this model can be fitted well to data. Specifically, we have implemented an optimization scheme that identifies optimal model parameters that give good fit 
between model volume curves and volume data in the atrium and ventricle during rest and pharmacological stress. We utilized patient-specific information to come up with initial model parameter values that served as initial guess in the optimization routine. The model was able to capture the blood flow dynamics of 20 young (half of which are women) and 20 elderly (half of which are women) subjects during rest, inotropic stress (dobutamine), and chronotropic stress (glycopyrrolate). We have quantified parameter uncertainty and verified the reliability of the parameter estimates through bootstrapping. Finally, a statistical test comparing the mean values of optimal parameters for the young vs the elderly and women vs men revealed that the model is able to give insight in age and sex related differences in heart functions. Also, a statistical test comparing the mean values of optimal parameters during rest and pharmacological stress displayed and quantified how the patients responded to the drugs.

\section{Acknowledgements}

This study was made possible through the Alternate Study Grant of the University of the Philippines and support of Roskilde University and the University of the Philippines Baguio. We would especially like to thank Philip Vingaard for the a priori estimates and preliminary investigations and Prof. Gilbert Peralta and Prof. Aurelio de los Reyes V for their interest in this work and for helpful discussions. 


\section{References}

Aboelkassem, Y., Savic, D., Campbell, S., 2015. Mathematical modeling of aortic valvce dynamics during systole. Journal of Theoretical Biology 365, 280-288.

Ahtarovski, K., Iversen, K., Lønborg, J., Madsen, P., Engstrøm, T., Vejlstrup., N., 2012. Left atrial and ventricular function during dobutamine and glycopyrrolate stress in healthy young and elderly as evaluated by cardiac magnetic resonance. American Journal of Physiology 303, H1469H1473.

Arbab-Zadeh, A., Dijk, E., Prasad, A., Fu, Q., Torres, P., Zhang, R., Thomas, J., Palmer, D., Levine, B., 2004. Effect of aging and physical activity on left ventricular compliance. Circulation 110, 1805-1811.

Aurigemma, G., Gottdiener, J., Arnold, A., Chinali, M., Hill, J., Kitzman, D., 2009. Left atrial volume and geometry in healthy aging: The cardiovascular health study. Circulation Cardiovascular Imaging 2, 282-289.

Blume, G., Mcleod, C., Barnes, M., Sewar, J., Pellikka, P., Bastiansen, P., Tsang, T., 2011. Left atrial function: Physiology, assessment, and clinical implications. European Journal of Echocardiography 12, 421-430.

Boyd, E., 1935. The Growth of the Surface Area of the Human Body. Oxford University Press.

Calderon, P., Habib, M., Kappel, F., de los Reyes V, A., 2017. Control aspects of the human cardiovascular-respiratory system under a nonconstant workload. Mathematical Biosciences 289, 142-152. 
Chowell, G., 2017. Fitting dynamic models to epidemic outbreaks with quantified uncertainty: A primer for parameter uncertainty, identifiability, and forecasts. Infectious Disease Modelling 2, 379-398.

Danielsen, M., 1998. Modeling of feedback mechanisms which control the heart function in a view to an implementation in cardiovascular models. IMFUFA tekst nr. .

Danielsen, M., Ottesen, J., 1997. A dynamical approach to the baroreceptor regulation of the cardiovascular system. Proceedings to the 5th International Symposium, Symbiosis '97, 25-29.

Douglas, P., 2003. The left atrium: a biomarker of chronic diastolic dysfunction and cardiovascular disease risk. Journal of the American College of Cardiology 42, 1206-1207.

Ellwein, L., Pope, S.R., Xie, A., Batzel, J., Kelley, C., Olufsen, M., 2009. Modeling cardiovascular and respiratory dynamics in congestive heart failure. Preprint .

Fan, H.H., Khoo, M., 2002. Pneuma a comprehensive cardiorespiratory model. Proceedings 2nd Joint EMBS/BMES Conference, 1533-34.

Field, A., 2013. Discovering Statistics with IBM SPSS. Newbury Park, CA: Sage.

Fujimoto, N., Hastings, J., Bhella, P., Shibata, S., Gandhi, N., CarrickRanson, G., Palmer, D., Levine, B., 2012. Effect of ageing on left ventricular compliance and distensibility in healthy sedentary humans. Journal of Physiology 590.8, 1871-1880. 
Grimes, R., Levine, R., Walker, P., Yoganathan, A., 1995. Dynamics of systolic pulmonary venous flow in mitral regurgitation: Mathematical modeling of the pulmonary venous system and atrium. Journal of the American Society of Echocardiography 8, 631-42.

Guyton, A., Hall, J., 2006. Textbook of medical physiology, 11th ed. Elsevier Inc. .

Guyton, A., Hall, J., 2016. Textbook of medical physiology, 13th ed. Elsevier Inc. .

Hayward, C., Kalnins, W., Kelly, R., 2001. Gender-related differences in left ventricular chamber function. Cardiovascular Research 49, 340-350.

Hoppensteadt, F., Peskin, C., 2002. Modeling and Simulation in Medicine and the Life Sciences. Springer-Verlag New York.

Howell, D., 2002. Statistical Methods for Psychology (5th ed.). Pacific Grove CA: Duxbury.

https://www.medicines.org.uk/emc/product/6270, . Electronic medicines compendium. URL: https://www . medicines . org. uk/emc/product/6270.

Kelley, C., 1999. Iterative Methods for Optimization. SIAM, Philadelphia, PA.

Lane, A., Ranadive, S., Yan, H., Kappus, R., Cook, M., Sun, P., Woods, J., Wilund, K., Fernhall, B., 2013. Effect of sex on wasted left ventricular effort following maximal exercise. Int J Sports Med 34, 770-776. 
Lim, E., Chan, G., Dokos, S., Ng, S., Latif, et al., L., 2013. A cardiovascular mathematical model of graded head-up tilt. PLoS ONE 8, e77357.

Lu, K., Clark, J., Ghorbel, F., Ware, D., Bidani, A., 2001. A human cardiopulmonary system model applied to the analysis of the valsalva maneuver. American Journal of Physiology 281, H2661-79.

Marquis, A., Arnold, A., Dean-Bernhoft, C., Carlson, B., Olufsen, M., 2018. Practical identifiability and uncertainty quantification of a pulsatile cardiovascular model. Mathematical Biosciences 304, 9-24.

Marwick, T., 2003. Stress echocardiography. Heart 89, 113-118.

Mcqueen, M., Peskin, C., 1989. A three-dimensional computational method for blood flow in the heart. ii. contractile fibers. Journal of Computational Physics 82, 289-297.

Najjar, S., Schulman, S., Gerstenblith, G., Fleg, J., Kass, D., O'Connor, F., Becker, L., Lakatta, E., 2004. Age and gender affect ventricular-vascular coupling during aerobic exercise. Journal of the American College of Cardiology 44, 611-617.

Olufsen, M., Ottesen, J., 2013. A practical approach to parameter estimation applied to model predicting heart rate regulation. Journal of Mathematical Biology 67, 39-68.

Olufsen, M., Ottesen, J., Tran, H., Ellwein, L., Lipsitz, L., Novak, V., 2005. Blood pressure and blood flow variation during postural change from sitting to standing: model development and validation. Journal of Applied Physiology 99, 1523-1537. 
Ottesen, J., 1997. Modelling of the baroreflex-feedback mechanism with time-delay. Journal of Mathematical Biology 36, 41-63.

Ottesen, J., 2011a. The mathematical microscope - making the inaccessible accessible. in "Betasys, Systems Biology of Regulated Exocytosis in Pancreatic B-cells" (eds. B. Boob-Bavnbek et al.), Springer Science+Business Media, LLC .

Ottesen, J., 2011b. Patient specific modeling of the heart as a tool for early diagnoses and treatment planning. ECMTB .

Ottesen, J., Danielsen, M., 2003. Modeling ventricular contraction with heart rate changes. Journal of Theoretical Biology 222, 337-346.

Ottesen, J., Olufsen, M., 2011. Functionality of the baroreceptor nerves in heart rate regulation. Comput. Methods Programs Biomed. 101, 208-219.

Peskin, C., Mcqueen, M., 1989. A three-dimensional computational method for blood flow in the heart i. immersed elastic fibers in viscous incompressible fluid. Journal of Computational Physics 81, 372-405.

Pope, S., Ellwein, L., Zapata, C., Novak, V., Kelley, C., Olufsen, M., 2011. Estimation and identification of parameters in a lumped cerebrovascular model. Mathematical Biosciences and Engineering 6, 93-115.

Redfield, M., Jacobsen, S., Borlaug, B., Rodeheffer, R., Kass, D., 2005. Age- and gender-related ventricular-vascular stiffening: a communitybased study. Circulation 112, 2254-2262. 
de los Reyes, A., Jung, E., Kappel, F., 2014. Stabilizing control for a pulsatile cardiovascular mathematical model. Bulletin of Mathematical Biology 76, $1306-1332$.

Rostagno, C., 2019. Heart valve disease in elderly. World Journal of Cardiology 11, 71-83.

Shoemaker, W., 1989. Fluids and electrolytes in the acutely ill adult. in "Textbook of Critical Care" (eds. W. Shoemaker S. Ayres, A Grenvik, P. Holbrook and W. Leigh Thompson), W. B. Saunders, Philadelphia .

Suga, H., Sagawa, H.and Shoukas, A., 1973. Load independence of the instantaneous pressure-volume ratio of the canine left ventricle and effects of epinephrine and heart rate on the ratio. Circulation Research 32, 314-322.

The Mathworks, Inc., . https://www.mathworks.com/help/index.html. URL: https : //www . mathworks . com/help/index .html.

Travin, M., Wexler, J., 1999. Pharmacological stress testing. Seminars in Nuclear Medicine 29, 298-318.

Ursino, M., 1998. Interaction between carotid baroregulation and the pulsating heart: a mathematical model. Am. J. Physiol. 275, H1733-H1747.

Vieira, M., Teixeira, R., Gonçalves, L., Gersh, B., 2014. Left atrial mechanics: echocardiographic assessment and clinical implications. Journal of the American Society of Echocardiography 27, 463-478.

Vingaard, P., Ottesen, J., 2017. Patientspecifik Modellering af Hjertet med 
Henblik på Diagnosticering af Hjertesvigt (Available upon request to the authors). Master's thesis. Roskilde Universitet.

WHO, . World health organization. URL: https://www. who. int/news-room/fact-sheets/detail/the-top-10-causes-of-death.

Williams, N., Brady, R., Gilmore, S., Gremaud, P., Tran, H., Ottesen, J., Mehlsen, J., Olufsen, M., 2019. Cardiovascular dynamics during head-up tilt assessed via pulsatile and non-pulsatile models. Journal of Mathematical Biology 79, 987-1014. 